\title{
Structure-Activity Relationships for a Large Diverse Set of Natural, Synthetic, and Environmental Estrogens
}

\author{
Hong Fang, ${ }^{\dagger}$ Weida Tong, ${ }^{*}, \dagger$ Leming M. Shi, ${ }^{\dagger, \ddagger}$ Robert Blair ${ }^{\S}$ Roger Perkins, ${ }^{\dagger}$ \\ William Branham, ${ }^{\S}$ Bruce S. Hass, ${ }^{\S}$ Qian Xie, ${ }^{\dagger}$ Stacy L. Dial, \\ Carrie L. Moland, ${ }^{\S}$ and Daniel M. Sheehan ${ }^{\S}$
}

\begin{abstract}
R.O.W. Sciences, Inc., 3900 NCTR Road, MC 910, J efferson, Arkansas 72079, and
Division of Genetic and Reproductive Toxicology, National Center for Toxicological Research, J efferson, Arkansas 72079
\end{abstract}

Received October 3, 2000

\begin{abstract}
Understanding structural requirements for a chemical to exhibit estrogen receptor (ER) binding has been important in various fields. This knowledge has been directly and indirectly applied to design drugs for human estrogen replacement therapy, and to identify estrogenic endocrine disruptors. This paper reports structure-activity relationships (SARs) based on a total of 230 chemicals, including both natural and xenoestrogens. Activities were generated using a validated ER competitive binding assay, which covers a $10^{6}$-fold range. This study is focused on identification of structural commonalities among diverse ER ligands. It provides an overall picture of how xenoestrogens structurally resemble endogenous $17 \beta$-estradiol $\left(E_{2}\right)$ and the synthetic estrogen diethylstilbestrol (DES). On the basis of SAR analysis, five distinguishing criteria were found to be essential for xenoestrogen activity, using $E_{2}$ as a template: (1) $\mathrm{H}$-bonding ability of the phenolic ring mimicking the $3-\mathrm{OH},(2) \mathrm{H}$-bond donor mimicking the17 $\beta-\mathrm{OH}$ and $\mathrm{O}-\mathrm{O}$ distance between 3- and $17 \beta-\mathrm{OH},(3)$ precise steric hydrophobic centers mimicking steric $7 \alpha$ - and $11 \beta$-substituents, (4) hydrophobicity, and (5) a ring structure. The 3-position $\mathrm{H}$-bonding ability of phenols is a significant requirement for $\mathrm{ER}$ binding. This contributes as both a H-bond donor and acceptor, al though predominantly as a donor. However, the $17 \beta-\mathrm{OH}$ contributes as a $\mathrm{H}$-bond donor only. The precise space (the size and orientation) of steric hydrophobic bulk groups is as important as a $17 \beta-\mathrm{OH}$. Where a direct comparison can be made, strong estrogens tend to be more hydrophobic. A rigid ring structure favors $E R$ binding. The knowledge derived from this study is rationalized into a set of hierarchical rules that will be useful in guidance for identification of potential estrogens.
\end{abstract}

\section{Introduction}

There is a growing body of evidence that some manmade chemicals, now called endocrine-disrupting chemicals (EDCs), ${ }^{1}$ have the potential to disrupt the endocrine system by mimicking endogenous hormones such as estrogens and androgens (1). Recent legislation mandates that the Environmental Protection Agency develop a screening and testing program for potential EDCs, of which xenoestrogens figure predomi nately (2). Xenoestrogens contain a number of chemical classes that display a broad range of structural diversity (3). For example, DES, DDTs, polychlorinated biphenyls (PCBs), alkylphenols, phthalates, and parabens have been found to be estrogenic. It has Iong been an enigma why such struc-

* To whom correspondence should be addressed: R.O.W. Sciences, Inc., 3900 NCTR Road, MC 910, J efferson, AR 72079. Telephone: (870) 543-7142. Fax: (870) 543-7382. E-mail: wtong@nctr.fda.gov.

† R.O.W. Sciences, Inc.

₹ Current address: BASF Corp., P.O. Box 400, Princeton, NJ 08543.

$\S$ National Center for Toxicological Research.

${ }^{1}$ Abbreviations: SAR, structure-activity relationship; QSAR, quantitative structure-activity relationship; $E R$, estrogen receptor; $h E R \alpha$, human estrogen receptor $\alpha$ subtype; EDCs, endocrine disrupting chemicals; RBA, relative binding affinity; NA, not active; NCTR, National Center for Toxicological Research; 2D, two-dimensional; 3D, three-dimensional; $\mathrm{E}_{2}, 17 \beta$-estradiol; $\mathrm{E}_{1}$, estrone; $\mathrm{E}_{3}$, estriol; $\mathrm{EE}$ ethynylestradiol; DES, diethylstilbestrol; DMS, dimethylstilbestrol; DDTs, 1,1,1-trichloro-2,2'-bischlorophenylethane derivatives; PCBs, polychlorinated biphenyls; log P, hydrophobicity. turally diverse chemicals have estrogenic activity like the endogenous hormone estradiol.

SARs for estrogens date back more than six decades to the early work of Dodds et al. $(4,5)$. The succeeding two decades saw the discovery of nonsteroidal estrogens, such as DES, based on understanding of the important structural features governing potency for steroidal estrogens. Recently, a number of SAR studies have been reported for steroidal estrogens (6) and nonsteroidal estrogens (7). These are generally focused on identification of structural characteristics for chemicals within similar two-dimensional (2D) structural frameworks, including $\mathrm{E}_{2}$ derivatives (6), DES derivatives (8), PCBS (9), phytoestrogens (10), al kylphenols (11), raloxifenes (12), and others. Modern computer-based tools have enabled the development of quantitative structureactivity relationship (QSAR) models for identifying steric and electrostatic features of a molecule in three-dimensional (3D) space for estrogenic activity (8, 13-19). Recent crystallographic structures of the human ER $\alpha$ subtype $(h E R \alpha)$ with a number of ligands, including $E_{2}$, DES, raloxifene, and 4-OH-tamoxifene, have also been reported $(20,21)$. By aligning these four ligands on the basis of the superposition of their ER binding sites, we have been able to demonstrate the common binding characteristics among these ligands (22). 
In principle, chemicals with similar biological activity share common structural features. This implies that structurally diverse estrogens possess a certain degree of structural commonality essential to eliciting estrogenic activity. Although a number of chemical classes are known to be estrogenic, little thorough structural evaluation has been presented for their structural resemblance to the $E_{2}$ or the strong synthetic estrogen DES. Moreover, only limited efforts have explored the structural similarities between different chemical classes of xenoestrogens. To better understand the structural requirements for $E R$ binding, it is important to have a reliable data set, obtained with consistent assay design, covering a broad range of chemical classes. Recently, we reported ER binding activity data for a large number of chemicals, including natural, synthetic, and environmental estrogens, using a validated rat ER competitive binding assay $(23,24)$. This data set, called the NCTR data set, currently contains 230 chemicals. It was designed a priori to cover broad structural diversity and a wide range of binding activities for elucidating the structural characteristics of xenoestrogens and natural estrogens.

The rat uterine cytosol ER competitive binding assay is the gold standard for in vitro ER assays. When our results are compared to results from other ER binding assays, there is a general consistency between relative ER activities across different assay methods and species (25). For example, we found a high linear correlation for ER binding affinities among a diverse group of chemicals assayed with the ER from rat uterine cytosol and $h E R \alpha{ }^{2}$ Further, we also found that ER assay results correlated very well with those from a yeast-based reporter gene assay and a MCF-7 cell proliferation assay. These findings demonstrate that $\mathrm{ER}$ binding is the major determinant across three levels of biological complexity (receptor binding, a yeast reporter gene response, and cell proliferation) of estrogen action. Moreover, chemicals positive in uterotrophic responses (in vivo estrogenic activity) are also positive in the ER binding assay, indicating that binding affinity is a good predictor of in vivo activity with few false negatives observed (26). Therefore, understanding the structural requirements for ER binding provides strong guidance in identifying potential in vivo estrogenic EDCs.

The NCTR data set covers most known estrogenic chemical classes as well as some new estrogenic chemicals. A careful SAR examination of the NCTR data set in conjunction with knowledge of the recently reported ligand-ER crystal structures should allow a better understanding of the general structural requirements of a chemical binding to the ER in a quantitative manner. In turn, the knowledge can be used to develop a predictive toxicology model for rapidly identifying potential estrogenic EDCs.

\section{Materials and Methods}

ER Binding Activities. The ER binding affinities of chemicals were determined by using a competitive receptor binding assay described previously (23). Briefly, a chemical's binding activity was determined by competing with radiolabeled [ $\left.{ }^{3} \mathrm{H}\right]-$ $\mathrm{E}_{2}$ for the ER in rat uterine cytosol. The I $\mathrm{C}_{50}(50 \%$ inhibition of $\left[{ }^{3} \mathrm{H}^{2} \mathrm{E}_{2}\right.$ binding) for each competitor was determined. The relative binding affinity (RBA) for each competitor was calculated by dividing the $I C_{50}$ of $E_{2}$ by the $I C_{50}$ of the competitor

\footnotetext{
${ }^{2}$ H. Fang, W. Tong, and D. Sheehan, unpublished results.
}

and multiplying by $100\left(E_{2}\right.$ RBA $\left.=100\right)$. The validated assay incubation conditions were $20 \mathrm{~h}$ at $4{ }^{\circ} \mathrm{C}$ using $17 \mathrm{mg}$ of uterine tissue $/ \mathrm{mL}\left(\mathrm{B}_{\max }=0.22 \mathrm{nM}\right)$ with $1 \mathrm{nM}\left[{ }^{3} \mathrm{H}\right] \mathrm{E}_{2}$. The competing chemical concentrations ranged from $1 \mathrm{nM}$ to $1 \mathrm{mM}$. Chemicals that failed to compete for $\left.{ }^{3} \mathrm{H}\right] \mathrm{E}_{2}$ binding to the $E R$ were designated as "not active" (NA). Chemicals that exhibited binding, but did not reach $50 \%$ inhibition in the designed concentration range, were designated as "slight binders". All assays were repeated at least twice; the $\mathrm{IC}_{50}$ values of positive chemicals are the means of the replicate values. The standard deviation of I $C_{50}$ for each chemical was reported (23), and only the mean RBA value was used for this study. The purity of chemicals as well as their effect on RBAs was also studied by Blair et al. (23). The largest fold difference ( $\sim 10$-fold) was found for nonylphenol from different commercial sources due to the impurity of the sample.

Molecular Modeling. The crystal structures of $E_{2}$, DES, raloxifene, and 4-OH-tamoxifen bound to the $\mathrm{ER}$ were obtained from the Protein Data Bank (PDB) as entries 1A52, 3ERD, $1 E R R$, and $3 E R T$, respectively. The alignment of these four ligands, based on root-mean-square (RMS) fitting of their receptor coordinates, was performed using the Insightll software package (Molecular Simulations, Inc., San Diego, CA). Log P was calculated using the atom/fragment contribution method (27). Pharmacophore searching was performed with the CATALYST package (Molecular Simulations, Inc.). The energy differences for $E_{2}$ and DES between the conformation in their binding modes and that in the minimum conformation of the free ligands were calculated using the AM 1 model Hamiltonian of the AMPAC/MOPAC module in I nsightII (Molecular Simulations, I nc.). The atom-atom distance was also measured using InsightlI (Molecular Simulations, Inc.).

\section{Results}

We determined the ER RBA of 230 chemicals, of which 130 were active and 100 inactive. To the best of our knowledge, this is the largest published ER competitive binding data set. This NCTR data set has been extensively used to build and validate a series of computational models proposed for priority setting of potential estrogenic EDCs (22).

For the convenience of analysis and presentation, the NCTR data set is divided into seven major categories according to the chemical structural characteristics. The chemical names and structures, as well as RBA values, are shown in Figures $1-7$ for 130 active chemicals and a few of the selected inactive chemicals. Steroids are chemicals with a steroidal backbone. $E_{2}$ derivatives have a phenolic $A$ ring (Figure $1 A$ ), whereas the others lack a phenolic $A$ ring (Figure $1 B$ ). The common structural feature of DES-like chemicals is two benzene rings separated by two carbons that are connected by a double bond in DES derivatives (Figure 2A) and by a single bond in hexestrol derivatives (Figure 2B). In this group of chemicals, triphenylethylene derivatives (Figure $2 \mathrm{C}$ ) are structurally similar to DES derivatives, but have an additional phenyl group attached to the ethylene bridge group. Most synthetic antiestrogens contain this structural feature. Phytoestrogens contain four major structurally distinct chemical classes: flavonoids, coumestans, chalconoids, and mycoestrogens. Flavonoids are the largest class, containing flavones (Figure 3A), flavanones (Figure 3B), and isoflavones (Figure $3 C$ ). They share a benzene ring directly connected to a chromone or 4-chromanone. Coumestans (Figure 3D) contain the smallest number of chemicals, of which coumestrol is the most rigid and flat molecule of all the phytoestrogens used. In contrast, chal conoids (F igure 3E) are the most structurally flexible 
(A) Steroids with a phenolic A ring

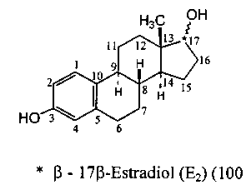

$\alpha-17 \alpha$-Estradiol $(3.07)$

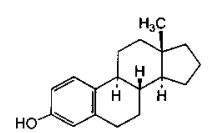

17.Deoxy-estradiol (14.1)

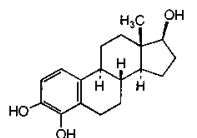

4-OH-Estradiol (52.9)

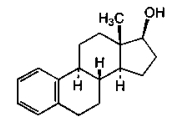

3-Deoxy-estradiol (0.50)

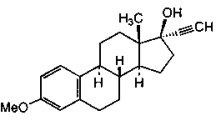

Mestranol (2.26)

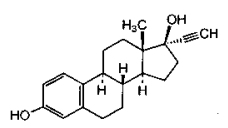

Ethynylestradiol (EE) (190)

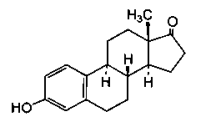

Estrone $\left(E_{1}\right)(7.31)$

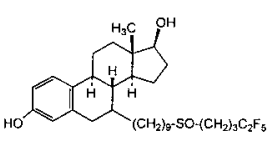

ICI $182,780(37.5)$

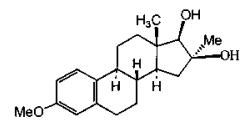

6ק-ol-16 $\alpha$-Methyl-3-methyl-estradiol (0.033)

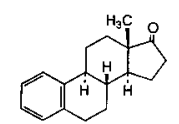

3-Deoxyestrone (0.006) Estra-1,3,5(10)-trien-16-one-3-ol (0.514)

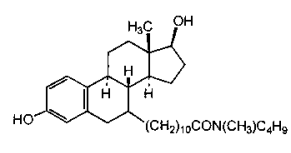

ICI $164,384(14.5)$
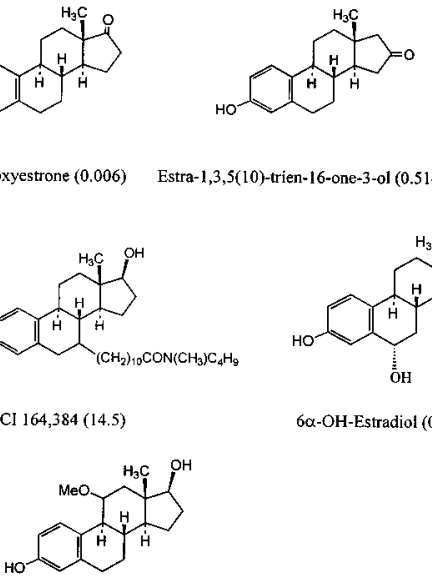

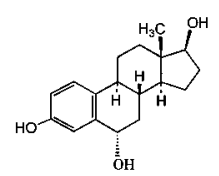

$6 \alpha$-OH-Estradiol (0.71)

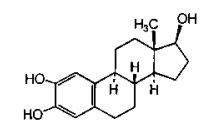

2-OH-Estradiol (22.4)

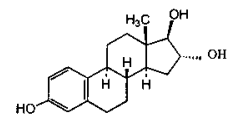

Estriol $\left(\mathbf{E}_{3}\right)(9.72)$

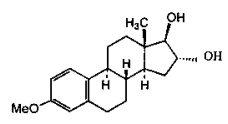

3-Methylestriol $(0.022)$

Figure 1. Steroids with (A) and without (B) a phenolic ring.

chemicals of the phytoestrogens. The generic structure of chalconoids is two benzene rings separated by three carbons. Mycoestrogens (Figure 3F) have a large ring fused with a benzene ring that contains two $\mathrm{OH}$ groups at positions 14 and 16 . Chemicals with two benzene rings connected by one carbon atom are classified as diphenylmethanes, including diphenolalkanes (Figure 4A), benzophenones (Figure 4B), and DDTs (Figure 4C). Biphenyls are chemicals with two phenyl rings directly connected to each other. They can be chlorinated as PCBs (Figure 5A) or nonchlorinated (Figure 5B). Alkyl phenols (Figure 6A), parabens (Figure 6B), and alkyloxyphenols (Figure $6 \mathrm{C}$ ) are categorized as phenols. They all contain a single phenolic ring. Most chemicals in this group have a long alkyl chain substituted at the para position. The chemicals that do not belong to any of these groups are found in miscellaneous (Figure 7).

Chemicals that exhibit ER binding have a broad range of structural diversity and RBAs. A common structural feature for steroids, DES-like chemicals, and most phytoestrogens is the presence of two rings (one of them usually a phenolic ring) separated by two carbons. Chemicals either with two rings separated by one carbon atom (diphenylmethanes), connected directly (PCBs), or possessing only one ring (alkyl phenols, phthalates, and kepone) typically have relatively lower binding affinities than chemicals with two rings separated by two atoms (25), although there is overlap in RBAs among some

(B) Steroids without a phenolic A ring

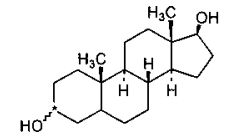

$* \alpha-3 \alpha$-Androstanediol (0.002)

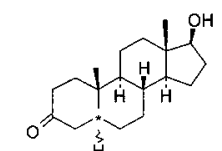
$* \alpha-5 \alpha-$ Dihydrotestosteron $(0.001)$
$\beta-5 \beta-$ Dihydrotestosteron (NA)

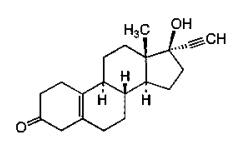

Norethynodrel (0.22)

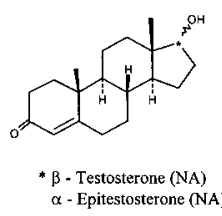

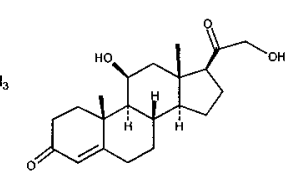

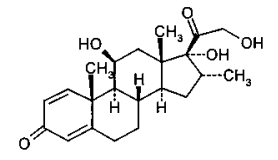

Cholesterol (NA)

Corticosterone (NA)

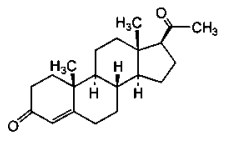

Progesterone (NA)

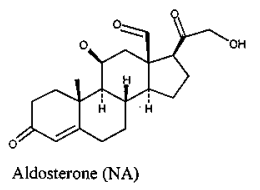

Aldosterone (NA)
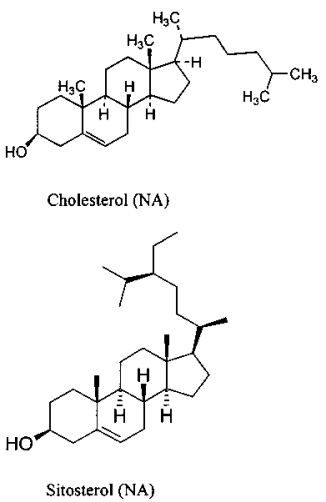

Dexamethasone (NA) chemical classes. Table 1 shows the mean RBA values for each chemical class. Steroids and DES-like chemicals have the strongest binding activities. The RBAs of the rest of the chemical classes follow this order: phytoestrogens $>$ diphenylmethanes $>$ biphenyls > phenols. There are only a few inactive chemicals in the DES-like chemical and phenol classes. The mean RBA of DES-like chemicals is 2.63, which is the highest of the groups that were examined, while phenols (mean RBA $=0.0015$ ) has the lowest mean RBA. These observations suggest that although both classes are likely to bind to the ER, their binding specificity is different due to the nature of their structures.

Steroids. $E_{2}$, with an RBA of 100 , is one of the most active estrogens. It has a hydrophobic backbone with $\mathrm{OH}$ groups at each end of the molecule. Such a molecular configuration was considered as the most important structural features for ER binding (6). The recently reported crystal structure of the ER-E $E_{2}$ complex reveals that the 3 - and $17 \beta-\mathrm{OH}$ groups primarily serve as $\mathrm{H}$-bond donors and acceptors in interacting with the receptor binding site (20). The elimination or modification of either of these two $\mathrm{OH}$ groups significantly reduces a chemical's binding affinity for the receptor, as shown in Table 2. This impact is more dramatic at the 3-position than at the $17 \beta$-position. For example, the loss of binding activity of 3-deoxy- $\mathrm{E}_{2}$ is 28-fold greater than the loss for 17-deoxy$\mathrm{E}_{2}$, indicating that the 3-OH is more important than the 
Table 1. General Information for the ER Ligand Categories, Including the Number of Chemicals with the Ratio of Active and Inactive Ligands, Mean RBA Values, ${ }^{a}$ and Representative Chemicals with Key Structural Featuresb

\begin{tabular}{|c|c|c|c|c|c|c|c|c|c|}
\hline \multirow{2}{*}{ ER Ligand Categories } & \multirow{2}{*}{$\begin{array}{l}\text { \# of } \\
\text { cmpds }\end{array}$} & \multirow{2}{*}{$\begin{array}{l}\text { Active / } \\
\text { Inactive }\end{array}$} & \multirow{2}{*}{$\begin{array}{l}\text { Mean } \\
\mathrm{RBA}^{a}\end{array}$} & \multirow{2}{*}{ Representative Chemicals (RBA) } & \multicolumn{5}{|c|}{ Key Structural Features $b$} \\
\hline & & & & & $\begin{array}{c}\text { Phenolic } \\
\text { Ring }\end{array}$ & $17 \beta-\mathrm{OH}$ & $\begin{array}{c}7 \alpha \text { or } 11 \beta \\
\text { steric bulks }\end{array}$ & $\begin{array}{l}\text { Additional } \\
\text { ring } c\end{array}$ & $\log \mathrm{P}$ \\
\hline DES-LIKE CHEMICALS & 20 & $19 / 1$ & 2.63 & DES (400) & 1 & 1 & 1 & 1 & 5.64 \\
\hline STEROIDS & 31 & $23 / 8$ & 2.45 & $E_{2}(100)$ & 1 & 1 & 0 & 1 & 3.94 \\
\hline PHYTOESTROGENS & 46 & $34 / 12$ & 0.069 & Genistein $(0.45)$ & 1 & 1 & 0 & 1 & 2.84 \\
\hline DIPHENYLMETHANES & 29 & $18 / 11$ & 0.01 & Bisphenol A (0.008) & 1 & 0 & 0 & 1 & 3.64 \\
\hline BIPHENYLS & 12 & $8 / 4$ & 0.0042 & $\begin{array}{l}\text { 2',3',4',5'-Tetrachloro- } \\
\text { 4-biphenylol }(0.23)\end{array}$ & 1 & 0 & 0 & 1 & 3.94 \\
\hline PHENOLS & 25 & $23 / 2$ & 0.0015 & Nonylphenol (0.03) & 1 & 0 & 0 & 0 & 6.0 \\
\hline
\end{tabular}

a Mean RBA values are calculated for active ligands only. ${ }^{b}$ The presence and absence of a structural feature are represented as 1 and 0 , respectively. ${ }^{C} A$ ring mimics one of the $B, C$, and $D$ rings of $E_{2}$.

Table 2. Effects of Elimination and Modification of the 3- and 17 $\beta$-OH on RBAs

\begin{tabular}{cclr}
\hline $\begin{array}{c}\text { elimination or } \\
\text { modification }\end{array}$ & $\begin{array}{c}\text { from } \\
\text { (RBA) }\end{array}$ & \multicolumn{1}{c}{$\begin{array}{c}\text { to } \\
\text { (RBA) }\end{array}$} & $\begin{array}{c}\text { fold } \\
\text { difference }\end{array}$ \\
\hline 3-OH & $E_{2}(100)$ & 3-deoxy- $E_{2}(0.5)$ & 200 \\
& $E_{1}(7.31)$ & 3-deoxy- $E_{1}(0.006)$ & 1218 \\
& $E(190)$ & mestranol $(2.26)$ & 84 \\
$17 \beta-\mathrm{OH}$ & $E_{3}(9.72)$ & 3-methylestriol & 442 \\
& $E_{2}(100)$ & 17-deoxy- $E_{2}(14.1)$ & 7 \\
& $E_{2}(100)$ & $E_{1}(7.31)$ & 14
\end{tabular}

$17 \beta-\mathrm{OH}$ in $\mathrm{ER}$ binding. This is consistent with the findings from the $\mathrm{E}-\mathrm{E}_{2}$ crystal structure that the $3-\mathrm{OH}$ has $\mathrm{H}$-bonding interactions with Glu 353, Arg 394, and a water molecule, whereas $17 \beta-\mathrm{OH}$ only forms one $\mathrm{H}$-bond with $\mathrm{His} 524$.

The elimination of 3-OH caused a greater reduction in the activity for estrone $\left(E_{1}\right)$ than for $E_{2}$ (Table 2). A similar result was also observed for $3-\mathrm{OH}$ methylation of estriol $\left(\mathrm{E}_{3}\right)$ and ethynylestradiol (EE). In both cases, the fold reduction in activity for stronger estrogens $\left(E_{2}\right.$ and $\mathrm{EE}$ ) was about 6 times lower than the fold reduction for relatively weak estrogens $\left(E_{1}\right.$ and $\left.E_{3}\right)$. This indicates that the phenolic ring is more critical for weak estrogens than strong ones.

The lack of a phenolic ring is a likely explanation for why most other steroids such as androgens, progesterones, and cholesterols were inactive in ER binding, or exhibited very low activity, such as dihydrotestosterone (Figure 1B). Clearly, the precise distance $\left(\mathrm{d}_{\mathrm{O}-\mathrm{O}}\right)$ between the 3- and $17 \beta-\mathrm{OH}$ groups of steroids, as well as their orientation, governs binding affinity. For examples, because the two $\mathrm{OH}$ groups had the same distance and orientation as those of $\mathrm{E}_{2}\left(\mathrm{~d}_{\mathrm{O}-\mathrm{O}}=11.0 \AA\right), 3 \beta$-androstanediol (RBA $=0.12, \mathrm{~d}_{\mathrm{o}-\mathrm{o}}=11.3 \AA$ ) was one of the most active steroids that does not contain a phenolic ring. Also, $17 \alpha-E_{2}\left(R B A=3.07, d_{0-0}=10.4 \AA\right)$ has a RBA 33-fold lower than that of $E_{2}$, and the RBA of $3 \alpha$-androstanediol $\left(\right.$ RBA $=0.002, \mathrm{~d}_{\mathrm{O}-\mathrm{O}}=9.95 \AA$ ) is 60 -fold lower than that of its $\beta$-isomer.

The RBA of 17-deoxy- $E_{2}(R B A=14.1)$ was slightly larger than that of estrone (RBA $=7.31$ ), indicating that the 17-ketone group might not contribute significantly to the binding as a $\mathrm{H}$-bond acceptor. If the ketone group of $E_{1}$ was moved from the 17-position to the 16-position to form estra-1,3,5(10)-trien-16-one-3-ol, the RBA was decreased 16-fold. A similar activity reduction was also observed with introduction of an $\mathrm{OH}$ group at the $16 \alpha-$ position of $E_{2}$, resulting in a relatively weak estrogen, $\mathrm{E}_{3}$. This demonstrates that a small polar group at the 16-position reduces a chemical's activity.

Systematic studies on the influences of substituents at various positions of $\mathrm{E}_{2}$ revealed that for most positions the introduction of substituents results in a loss of binding affinity $(17,28)$. For example, the $\mathrm{E}_{2}$ metabolites $2-\mathrm{OH}-\mathrm{E}_{2}(\mathrm{RBA}=22.4)$ and $4-\mathrm{OH}-\mathrm{E}_{2}(\mathrm{RBA}=52.9), 6 \alpha-$ $\mathrm{OH}-\mathrm{E}_{2}(\mathrm{RBA}=0.71)$, and $\mathrm{E}_{3}(\mathrm{RBA}=9.72)$ were less active than $E_{2}$ with 2-140-fold differences. The only positions where introduction of a substituent does not interfere with binding are $7 \alpha, 11 \beta$, and $17 \alpha$ (6). The degree of increase or decrease in activity is strongly dependent on the substituents in those positions. The $7 \alpha-$ and $11 \beta$-positions are structurally equivalent for steroids and can bear large substituents. Small steric substituents introduced at these positions generally increase activity (29). L arge substituents reduce the RBA and give rise to antiestrogenic activity, such as ICI 182,780 (RBA = 37.5) and ICI 164,384 (RBA = 14.5). A 17 $\alpha$-ethynyl substituent is favorable for $E R$ binding. $E E$ is a stronger estrogen $(R B A=190)$ than $E_{2}(R B A=100)$. Norethynodrel (RBA $=0.22$ ) is a progesterone derivative with a $17 \alpha$-ethynyl substitution, and is a popular oral contraceptive drug. It is a moderately strong binder (454-fold weaker than $E_{2}$ ).

DE S-like Chemicals. DES (RBA $=400)$ is one of the highest-affinity synthetic estrogens. I ts activity is 4 times 
(A) DES derivatives

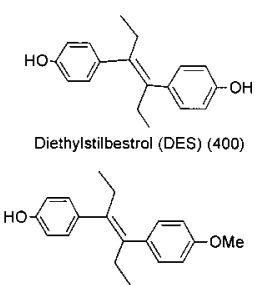

DES monomethyl ether (20.43)

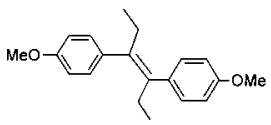

DES dimethyl ether $(0.056)$

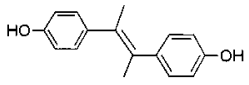

Dimethyistilbestrol (DMS) (14.50)

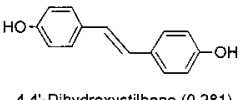

4,4'-Dihydroxystilbene $(0.281)$

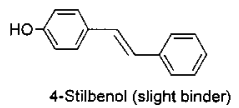

(B) Hexestrol derivatives

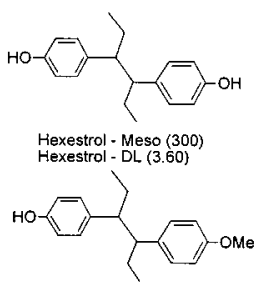

Hexestrol monomethyl ether $(9.37)$

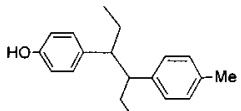

Meso-p-(a,b-diethyl-p-methylphenethyl)phenol (4.00)
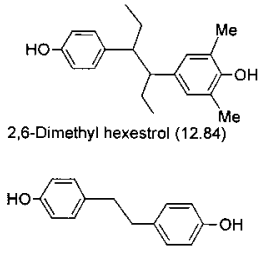

4,4'-Ethylenediphenol $(0.037)$

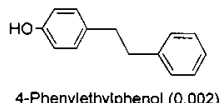

4-Phenylethylphenol (0.002)
(C) Triphenylethylenes
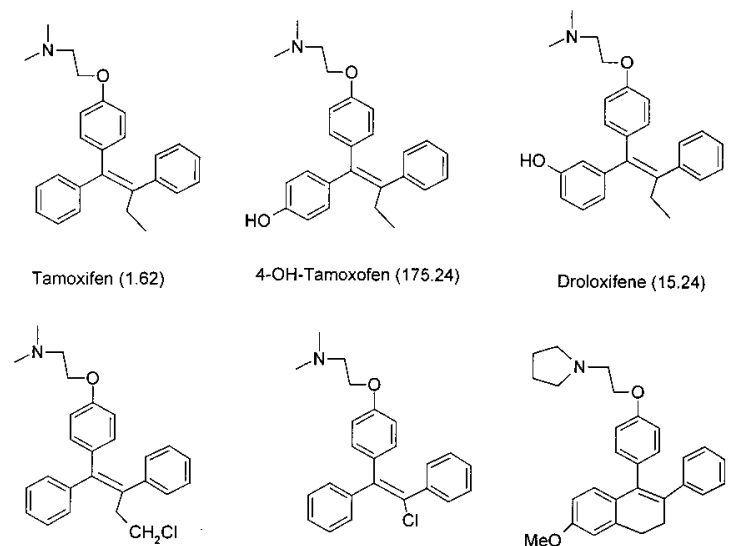

4-OH-Tamoxofen (175.24) Drolaxifene $(15.24)$

Toremifen $\{1.38)$

Clomiphene $(0.719)$

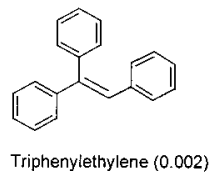

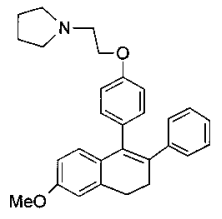

Nafoxidine (0.719)
Triphenylethylene $(0.002)$

Figure 2. (A) DES, (B) hexestrol, and (C) triphenylethylene derivatives.

higher than that of $E_{2}$. The symmetry of the molecule, the distance between the two $\mathrm{OH}$ groups, and the two ethyl side chains provide precisely correct spacing for hydrophobic and $\mathrm{H}$-bond interactions (21).

To efficiently bind to a receptor, the ligand tends to adopt a conformation that generally is not the one with the lowest energy. The free ligand which is in a stable conformation adjusts to a less stable conformation to achieve optimal binding. N evertheless, ligand binding to the ER will release energy in general. The energy difference of a mol ecule, between the conformation in its binding mode and that in the minimum conformation of the free ligand, contributes to the binding affinity in a

negative way. The less energy a molecule sacrifices during the binding, the stronger the binding affinity will be. $E_{2}$ has a much larger energy difference $\left(\Delta H_{f}=40.5\right.$ $\mathrm{kcal} / \mathrm{mol})$ than DES $\left(\Delta \mathrm{H}_{\mathrm{f}}=17.8 \mathrm{kcal} / \mathrm{mol}\right)$, which might hel $p$ explain why DES is a better binder.

The $\mathrm{O}-\mathrm{O}$ distance is $1 \AA$ larger in DES $\left(\mathrm{d}_{\mathrm{O}-\mathrm{O}}=12.1\right.$ $\AA$ ) than in $E_{2}$ and functionally resembles the 3 - and $17 \beta$ $\mathrm{OH}$ 's of $\mathrm{E}_{2}$. The loss of the H-bonding capability of one $\mathrm{OH}$ group reduces the RBA. For example, if one of the $\mathrm{OH}$ groups is converted to its methyl ether, DES monomethyl ether $(\mathrm{RBA}=20.43)$, the RBA decreases $\sim 20$ fold (Figure 2A). A similar activity reduction was also observed for hexestrol $(\mathrm{RBA}=300)$ (Figure $2 \mathrm{~B})$; replacing one of the $\mathrm{OH}$ groups with a methoxy group resulted in a 32-fold less active derivative, hexestrol monomethyl ether (RBA = 9.37). The reduction in activity is much more significant when both $\mathrm{OH}$ groups of DES are methylated; the RBA decreases more than 7000-fold for DES dimethyl ether (RBA $=0.056$ ). This demonstrates that while the loss of one $\mathrm{H}$-bond donor will reduce the binding affinity by $\sim 20$-fold, the loss of the second $\mathrm{H}$-bond donors diminishes the activity by a further 350 -fold.

4-Methyl-substituted hexestrol [meso-p-( $\alpha, \beta$-diethyl-pmethylphenethyl)phenol, RBA $=4.00$ ] and hexestrol monomethyl ether $(R B A=9.37)$ have comparable binding affinities. This is consistent with the observation that 17deoxy- $E_{2}$ has an RBA close to that of $E_{1}$. In both cases, the chemicals with or without a $\mathrm{H}$-bond acceptor exhibit similar RBAs. It suggests that with a phenolic ring at one end, the contribution of the $\mathrm{OH}$ group at the other end is mainly through $\mathrm{H}$-bond donor interaction with the receptor.

The degree of contributions to the binding activity of the two ethyl groups of DES is as significant as its $\mathrm{OH}$ groups. DES (RBA $=400)$, dimethylstilbestrol (DMS, RBA $=14.50)$, and 4,4'-dihydroxystilbene $(R B A=0.28)$ are in sequence one carbon atom shorter at the two ethylene side chains. The RBAs of these latter two chemicals were 28- and 1423-fold lower than that of DES. The ethyl groups may contribute to ER binding in three distinct ways. First, they increase the molecule's hydrophobicity. The hydrophobicity of DES ( $\log P=5.64)$ is larger than that of $E_{2}(\log P=3.94)$. The two ethyl groups contribute more than one-third of the hydrophobicity of the overall structure. Second, they maintain the rigid binding conformation of DES. The decrease in the length of the side chains introduces more flexibility for both phenolic rings. Third, probably the most important contribution of the ethyl groups to binding is to occupy the precise space that interacts with the receptor binding pocket site, which resembles the $11 \beta$ - and $7 \alpha$-substituents of $E_{2}$, and therefore increase its RBA. It is evident that the attachment of small alkyl substituents to the $11 \beta$ position can lead to a considerable increase in binding affinity for $\mathrm{E}_{2}$, such as $11 \beta$-chloromethyl-, ethyl-, and vinyl- $E_{2}(28)$ with RBA values in the range of $120-230$. On the basis of the recent crystal structure of the $E_{2}-$ and DES-ER complexes, both ligands could be aligned together by overlaying their receptor coordinates. By modifying the ethyl group to the $11 \beta$-position of the $\mathrm{E}_{2}$ binding conformation, we have been able to compare the relative position of the ethyl groups from $11 \beta$-ethyl- $E_{2}$ with that of DES. As shown in Figure 8, the ethyl groups from both molecules are positioned extremely close to each other, and fit well in the hydrophobic binding pocket. This observation also suggests that the specific 
(A) Flavones

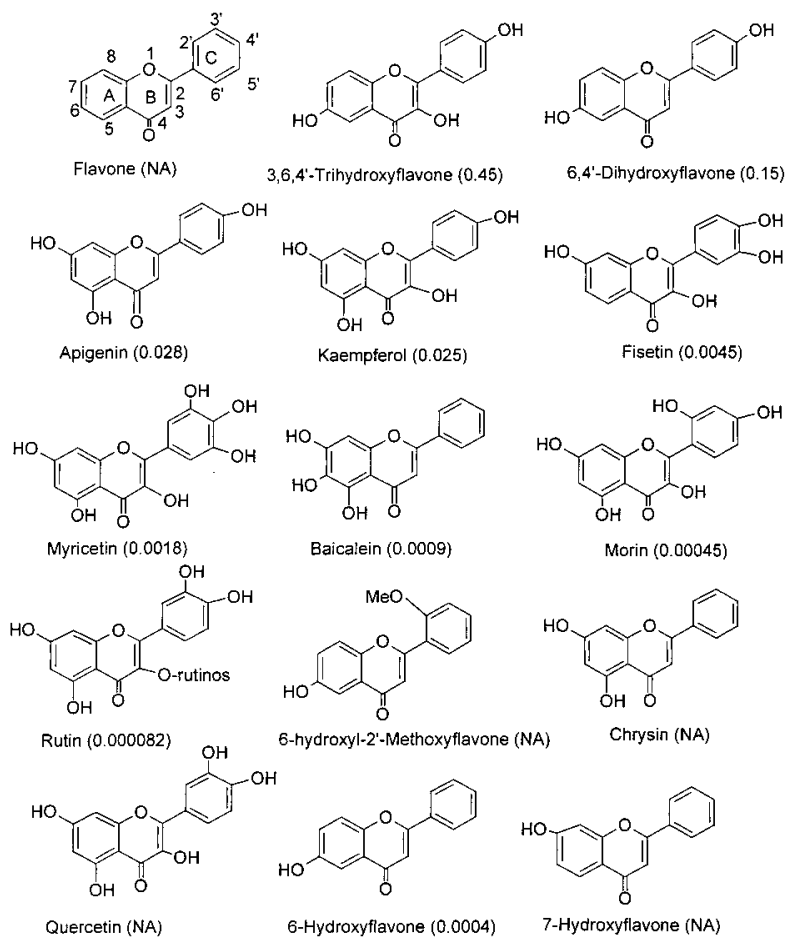

(B) Flavanones

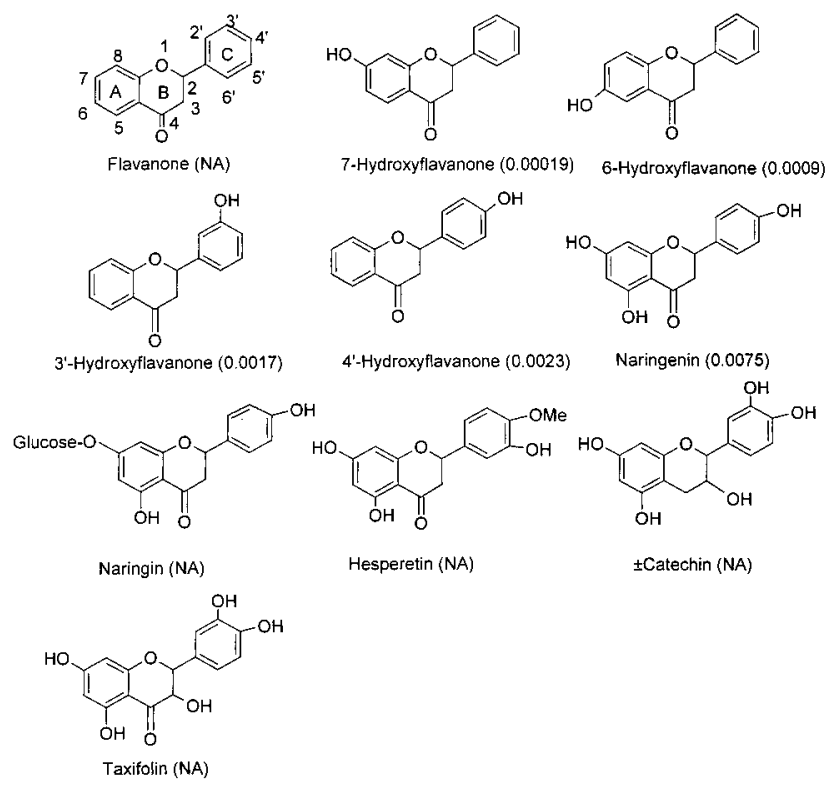

(1)

Genistein (0.45)
(C) Isoflavones
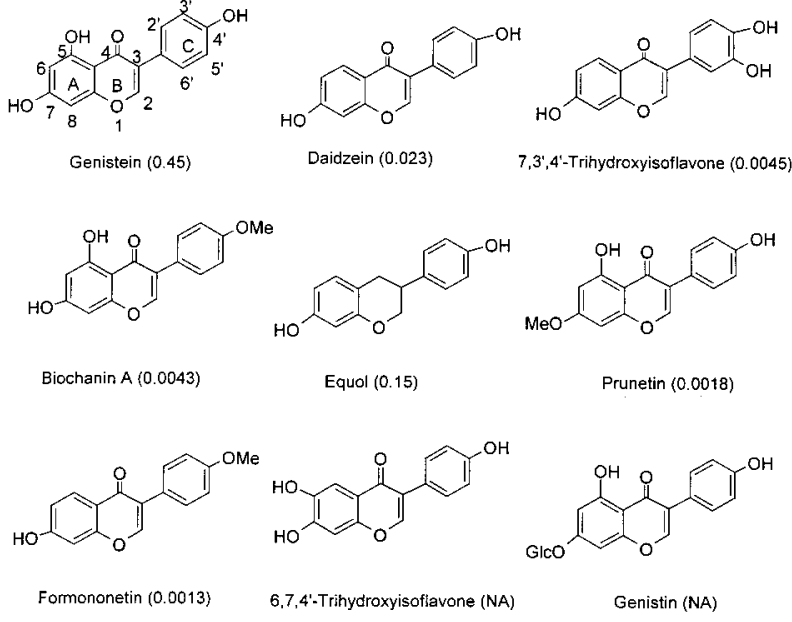

(D) Coumestans

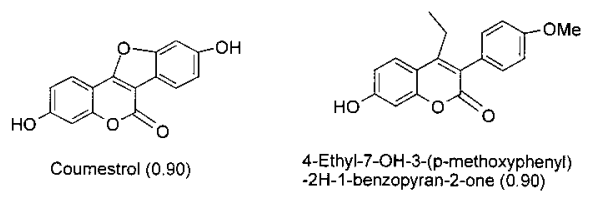

(E) Chalconoids

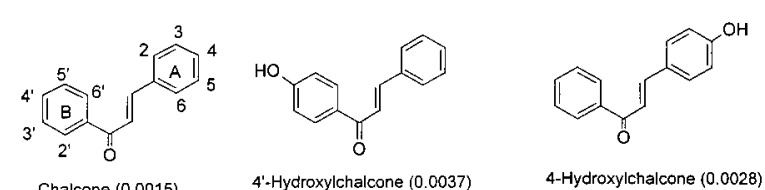<smiles>O=C(/C=C/c1ccc(O)cc1)c1ccc(O)cc1O</smiles>

4.2'4-Trihydroxychalcone $(0.054) \quad$ Phloretin $(0.069)$

(F) Mycoestrogens

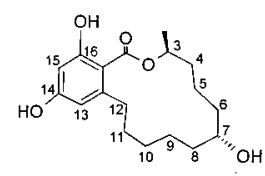

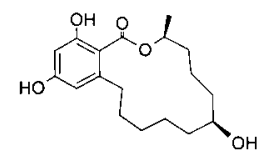

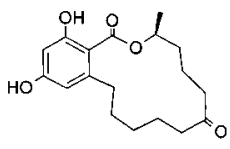

$\alpha$-Zearalanol (30)

ß-Zearalano: (0.64)

Zearalanone $\{2.1\}$<smiles>CCCC/C=C/c1cc(O)cc(O)c1C(=O)OC(C)CCCCCC(O)O</smiles>

$\alpha \cdot$ Zearalenol (43)

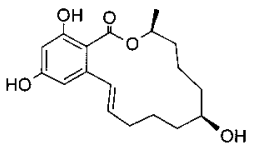

$\beta$-Zearalenol $(0.20)$

Figure 3. (A) Flavones, (B) flavanones, (C) isoflavones, (D) coumestans, (E) chalconoids, and (F) mycoestrogens.

orientation of the two ethyl groups of DES is critical to its function. That may explain why meso-hexestrol (RBA $=300)$ was 80-fold stronger than DL-hexestrol (RBA = 3.60).

In general, DES derivatives were stronger binders than hexestrol derivatives, because the former has a rigid double bond connecting the two benzene rings. This is evident from the RBA comparison of three pairs of chemicals between these two chemical classes: DES versus hexestrol, DES monoether versus hexestrol mono- ether, and 4,4'-dihydroxystilbene versus 4,4'-ethylenediphenol. However, 4-phenylethylphenol $(R B A=0.002)$ with single phenol group was more active than 4-stilbenol (slight binder). These observations indicate that when both $\mathrm{OH}$ groups contribute to the binding, a rigid structure is critical for a better fit to the ER. However, when a chemical contains only one phenolic ring, binding is dependent on how well the rest of the structure fits into the binding pocket and the binding activity is more favorable to a chemical with certain flexibility. 
(A) Diphenolalkanes

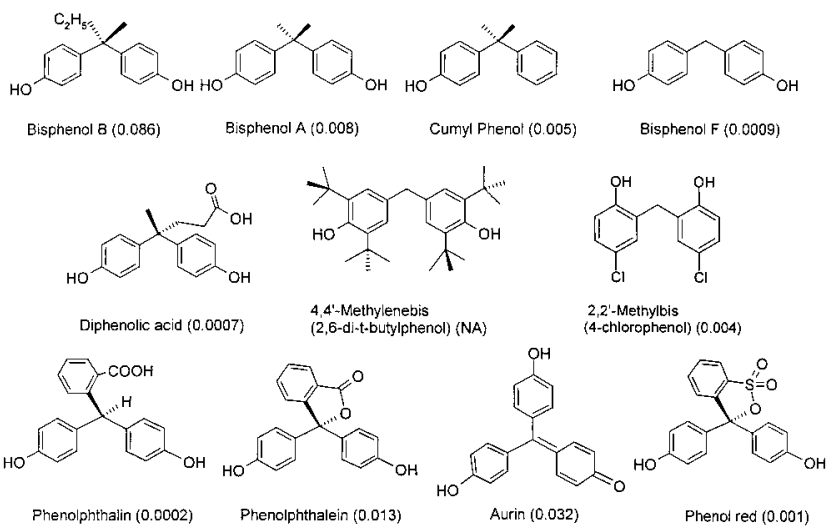

(B) Benzophenones

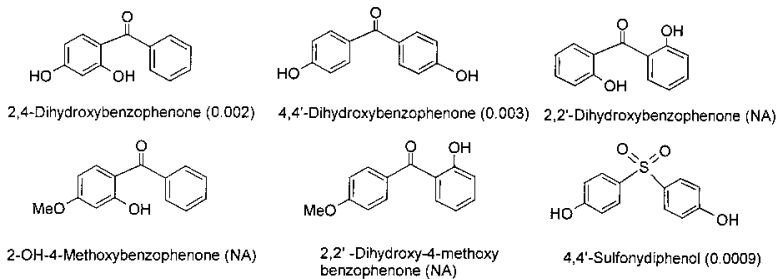

(C) DDTs
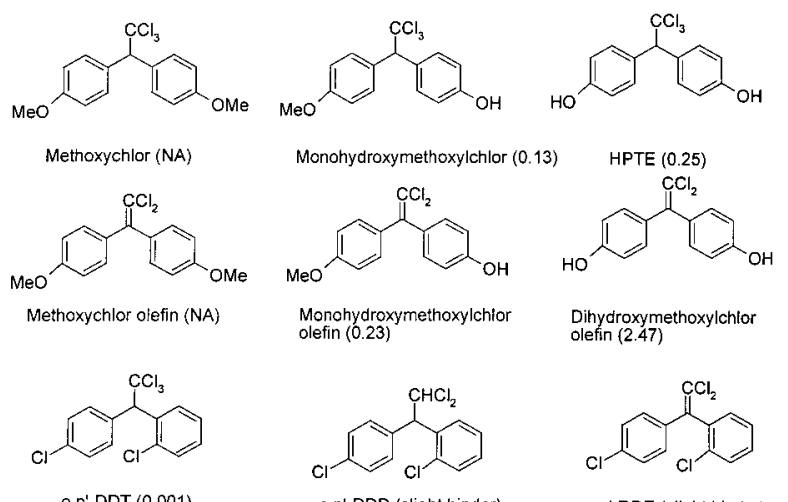

O.P'-DDT (0.001)

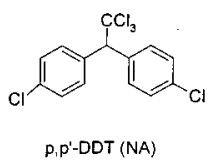

O,P'-DDD (slight binder)

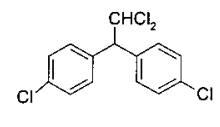

p.p'-DDD (NA)

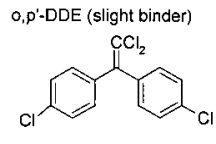

p,p'-DDE (NA)

Figure 4. (A) Diphenolalkanes, (B) benzophenones, and (C) DDTs.

Triphenylethylenes (Figure 2C), which act as antiestrogens, are structurally similar to DES, but have an additional phenyl group attached at the ethylene moiety. The RBA value, presented here, cannot distinguish agonist from antagonist activity. 4-OH-tamoxifen (RBA $=175.24$ ) binds to the ER 100 times stronger than tamoxifen (RBA $=1.62$ ) and 10 times stronger than droloxifene (RBA $=15.24)$ because its phenolic ring resembles the $A$ ring of $E_{2}$. If its $\mathrm{OH}$ group is eliminated (tamoxifen, RBA $=1.62$ ) or its position is changed (droloxifene, RBA $=15.24$ ), the binding affinity is re duced. For clomiphene, a chloro substituent at the ethylene and, for toremifene, a chloro substituent at the ethyl side chain both produce binding affinities similar to that of tamoxifen.

Phytoestrogens. There are four major structurally distinct chemical classes of phytoestrogens (10): fla-

(A) PCBs

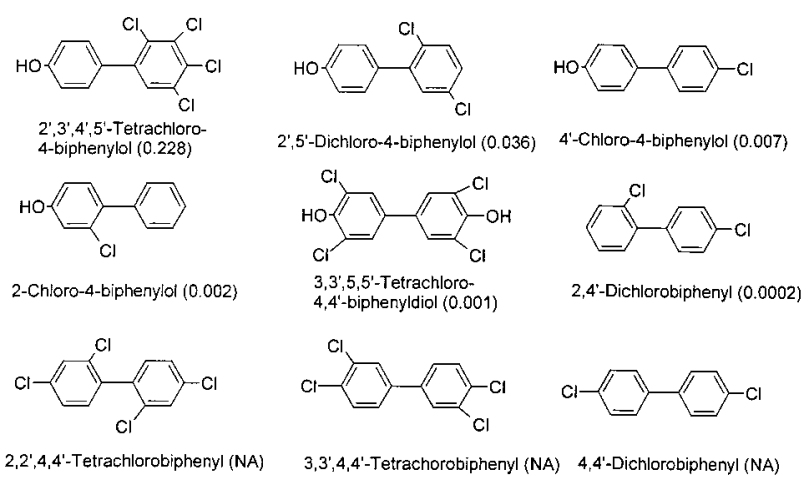

(B) Non-chlorinated biphenyls
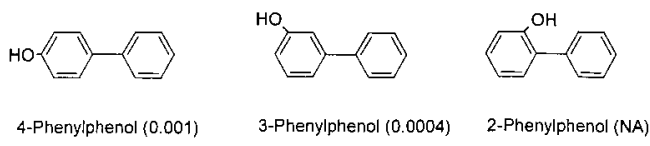

Figure 5. (A) PCBs and (B) nonchlorinated biphenyls.

vonoids, coumestans, chalconoids, and mycoestrogens. The binding activity of these four classes follows this order: mycoestrogens > coumestans > flavonoids > chalconoids. The representative chemicals for each class are $\alpha$-zearalenol (RBA $=43$ ), coumestrol (RBA $=0.9$ ), genistein (RBA $=0.45)$, and phloretin (RBA $=0.07$ ).

Flavonoids are the largest class of phytoestrogens, which include flavones (Figure $3 \mathrm{~A}$ ), flavanones (Figure 3B), and isoflavone (F igure $3 \mathrm{C}$ ). Their basic construction consists of a benzene ring directly connected to $\mathrm{C} 2$ or $\mathrm{C} 3$ of chromone or 4-chromanone. The positions of the A and $C$ benzene rings of flavonoids resemble those in DES, where they are separated by two atoms in a trans conformation. Thus, the binding affinity of flavonoids is largely dependent on the relative positions of the $\mathrm{OH}$ groups at the $A$ and $C$ rings, respectively, to mimic the $4,4^{\prime}-\mathrm{OH}$ of DES. It is generally considered that isoflavones are more active than flavones (30). That is because most isoflavones, such as genistein, equol, and daidzein, have two $\mathrm{OH}$ groups at the 7- and 4'-positions that match the 4- and 4'-positions of DES, respectively (Figure 3C). Although most flavones such as apigenin, kaempferol, fisetin, and morin also have the 7- and 4'-positions occupied by $\mathrm{OH}$ groups, these two positions correspond to the 3- and 4'-positions of DES (Figure 3A). Actually, the 6- and 4'-positions of flavones match the 4- and 4'positions of DES, respectively. This was evident because 3,6,4'-trihydroxyflavone (RBA $=0.45$ ) and 6,4'-dihydoxyflavone (RBA $=0.15$ ) have RBAs comparable to those of isoflavones.

Like the 4- and 4'-OH groups of DES, the 6- and 4'$\mathrm{OH}$ groups of flavonoids (7- and 4'-OH in isoflavones) play a similar role in binding. Although a small difference ( 2.5-fold) in RBA values was observed for the biochanin $A(R B A=0.0043)$ and prunetin $(R B A=0.0018)$ pair and the 6-hydroxyflavanone (RBA $=0.0009$ ) and 4'-hydroxyflavanone ( $R B A=0.0023$ ) pair, it is difficult to eliminate the possibility that both phenolic rings ( $A$ and $C$ ) of flavonoids can functionally mimic the phenolic $A$ ring of $E_{2}$. This suggests the possibility that flavonoids may bind to the $E R$ in either of two orientations that differ by $180^{\circ}$. The recent description of the crystal structure for the genistein-ER $\beta$ complex revealed that its phenolic $C$ ring 
(A) Alkylphenols
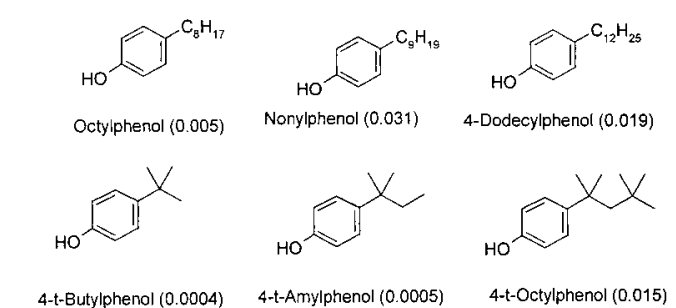

4-t-Butylphenol $(0.0004)$

4-t-Amylphenol $(0.0005)$

4-t-Octylphenol (0.015)
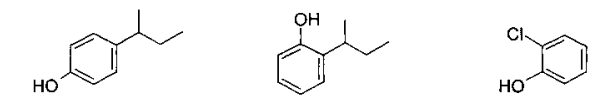

4-sec-Butylphenol $(0.00043)$
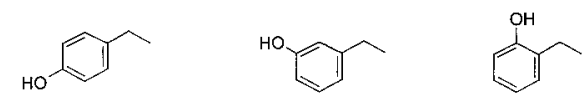

4-Ethylphenol $(0.00007)$

3-Ethylphenol (0.00014) 2-Ethylphenol $(<0.00001)$

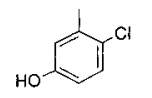

4-Chloro-3-methyl phenol $(0.00042)$
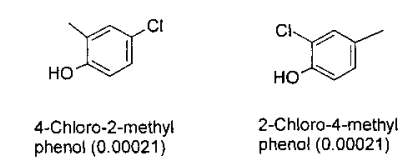
4-Chlorc-2-methy
phenol $(0.00021)$ phenol $(0.00021)$

(B) Parabens

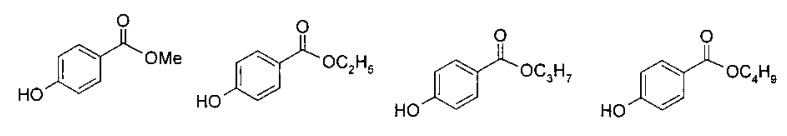

Methyl paraben (0.0004) Ethyl paraben (0.0006) Propyl paraben(0.0006) Butyl paraben (0.0009)

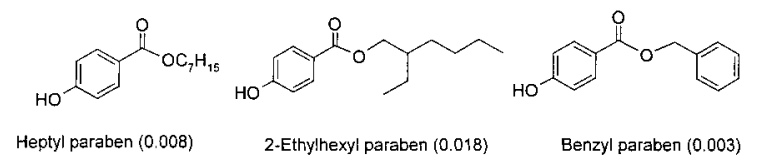

Heptyl paraben $(0.008)$

2-Ethylhexyl paraben $(0.018)$

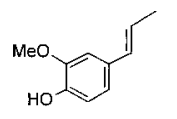

Isoeugenol (NA)
(C) Alkyloxyphenol
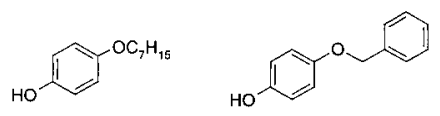

Figure 6. (A) Alkyl phenols, (B) parabens, and (C) alkyloxyphenols.

served the same function as the $A$ ring of $E_{2}$ to form the $\mathrm{H}$-bond interaction with the receptor (31).

Like DES derivatives that have larger RBAs than hexestrol derivatives, flavones are slightly better binders than flavanones because their benzene rings are attached to the double bond of the $B$ ring of chromone, which leads to a more rigid and flat structure. Within the same structural frame, the flavone apigenin $(\mathrm{RBA}=0.028$ ) has an RBA 4 times higher than that of the flavanone naringenin (RBA $=0.0075)$. Similarly, genistein was more potent than 2,3-dihydroxygenistein (32). However, the result is only applicable for flavonoids containing both $\mathrm{OH}$ groups at the $\mathrm{A}$ and $\mathrm{C}$ rings. The relative activity is reversed between 6-hydroxyflavanone (RBA $=0.0009$ ) and 6-hydroxyflavone (RBA $=0.0004)$, which are consistent with observations on DES-like chemicals.

Generally, a large steric hindrance will reduce activity. Genistein and naringin are inactive compared to genistein $(\mathrm{RBA}=0.45)$ and naringenin (RBA $=0.0075)$. However, compared to quercetin (NA), rutin exhibited a very low activity. This indicates that the 3-position of flavones is equivalent to the $7 \alpha$-position of $E_{2}$ where a large group can be fitted in the binding pocket.
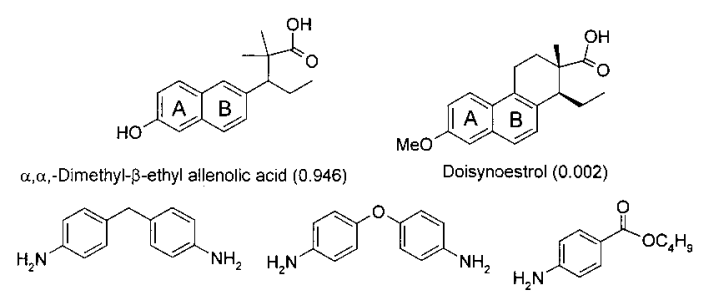

4,4'-Methylenedianiline (NA) 4-Aminophenyl ether (NA) Butyl-4-aminobenzoate (NA)
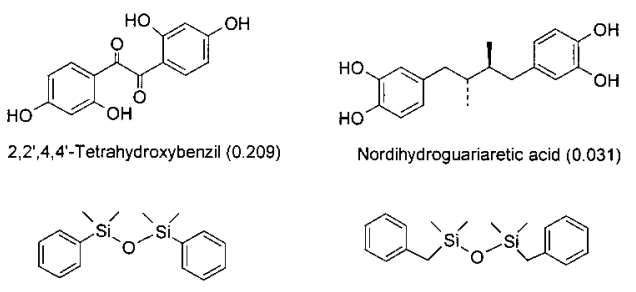

1,3-Diphenyltetramethyldisiloxarn (0.0007) 1,3-Dibenzyltetramethyddisiloxane (NA)
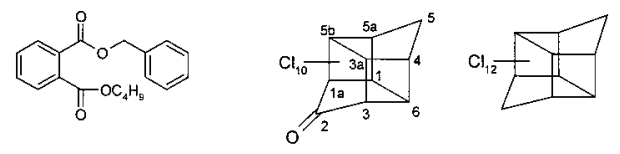

Butyibenzylphthalate (slight binder)

Kepone (0.013)

$\operatorname{Mirex}(\mathrm{NA})$

Figure 7. Miscellaneous chemicals.

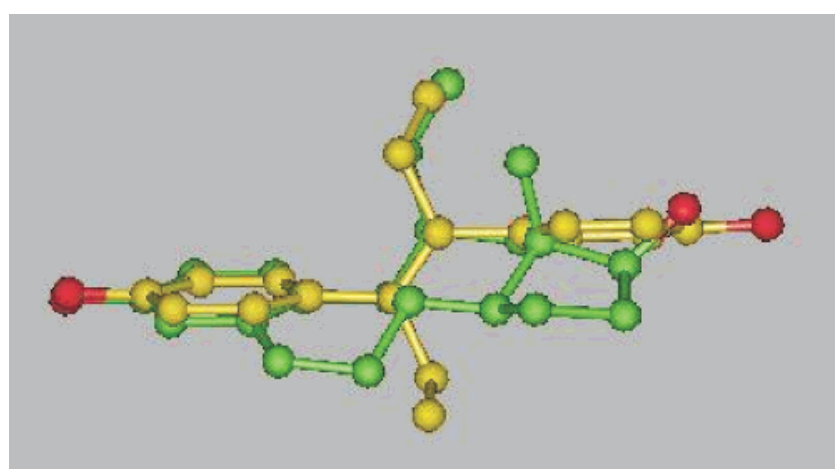

Figure 8. Superimposed structures of $11 \beta$-ethyl- $E_{2}$ (green) and DES (yellow). $E_{2}$ and DES are first aligned on the basis of the superposition of the receptor coordinates of the crystal structures between the $\mathrm{E}_{2}-$ and DES-ER complexes. The $11 \beta$ position of the $E_{2}$ is then modified by adding an ethyl group to form $11 \beta$-ethylestradiol. The ethyl groups of $11 \beta$-ethyl- $E_{2}$ and DES are positioned closely in a hydrophobic binding pocket.

Converting one of the $\mathrm{OH}$ groups to a methyl ether significantly reduced the RBA of isoflavones. Prunetin $(\mathrm{RBA}=0.0018)$ and biochanin $\mathrm{A}(\mathrm{RBA}=0.0043)$ have 250- and 100-fold lower RBAs, respectively, than their parent compound, genistein (RBA $=0.45$ ), while the RBA of formononetin (RBA $=0.0013)$ is 17 -fold lower than that of daizein.

Unlike the fact that $1-O H-E_{2}$ is less potent than $E_{2}$ (33), an additional $\mathrm{OH}$ group in position 5 of isoflavones, which resembles the 1-position of $E_{2}$, increases estrogenic activity $(10,34)$. Specifically, genistein is 20 -fold more active than daizein, and biochanin $\mathrm{A}$ is 4-fold more active than formononetin. This may be explained by the formation of an intramolecular $\mathrm{H}$-bond between the $5-\mathrm{OH}$ and the carbonyl groups which enhances the electron withdrawal of the carbonyl group and lead to a better 7-OH $\mathrm{H}$-bond donor.

Two coumestans were assayed, and both had RBAs $\sim 100$-fold lower than that of $E_{2}$ (Figure 3D). While coumestrol has a relatively rigid and flat structure that is similar to $E_{2}$, 4-ethyl-7-hydroxy-3-(p-methoxyphenyl)- 
Table 3. Relationship between the $\mathrm{O}-\mathrm{O}$ Distance and the RBA

\begin{tabular}{cccccc}
\hline mycoestrogen & RBA & $\mathrm{d}_{\mathrm{O}-\mathrm{O}}(\AA)$ & mycoestrogen & $\mathrm{RBA}$ & $\mathrm{d}_{\mathrm{O}-\mathrm{O}}(\AA)$ \\
\hline$\alpha$-zearalanol & 30 & 11.00 & $\beta$-zearalenol & 0.20 & 9.85 \\
$\alpha$-zearalenol & 43 & 11.28 & zearalanone & 2.1 & 9.77 \\
$\beta$-zearalanol & 0.64 & 9.23 & & &
\end{tabular}

$2 \mathrm{H}$-1-benzopyran-2-one has an ethyl group that is functionally similar to that of DES. The latter had the same RBA as coumestrol because its ethyl group favors binding which compensates for the reduced RBA form converting one of the $\mathrm{OH}$ groups to a methyl ether.

The generic structure of chalconoids is two benzene rings connected by three carbons (Figure $3 \mathrm{E}$ ). The $\mathrm{O}-\mathrm{O}$ distance between the two $\mathrm{OH}$ groups in the $\mathrm{B}$ ring and the one in the A ring is $\sim 11$ and $\sim 12 \AA$, respectively, which is comparable to those of DES and $E_{2}$. Since their flexible molecular structures do not favor binding, chalconoids have RBAs that are 1000 -fold lower than that of $\mathrm{E}_{2}$. The elimination of one of the $\mathrm{OH}$ groups on one side, such as 4-hydroxychalcone and 4'-hydroxychalcone, caused a loss in activity of $\sim 20$-fold. The results further demonstrate that two $\mathrm{OH}$ groups within a precise distance play an important role in ER binding.

Mycoestrogens are the most active chemi cals in phytoestrogens (F igure 3F). As shown in Table 3, $\alpha$-zearalenol and $\alpha$-zearalanol had RBAs 2 orders of magnitude higher than those of their $\beta$-isomers. The activity of zearalanone was between those of its $\alpha$ - and $\beta$-isomers. The order of their RBAs was consistent with that of their $\mathrm{O}-\mathrm{O}$ distances. In $\alpha$-zearalenol and $\alpha$-zearalanol, these were about $11.0 \AA$, the same distance found between the 3and $17 \beta-\mathrm{OH}$ groups of $\mathrm{E}_{2} ; \beta$-zearalanol and $\beta$-zeralenol are $1 \AA$ shorter (Table 3). This indicates that their RBAs are dependent on one of the critical structural features, the $\mathrm{O}-\mathrm{O}$ distance, when chemicals have the same structural frameworks.

Diphenylmethanes. The generic structure for this chemical class is two benzene rings separated by one carbon (or other atoms) (Figure 4). The 4-OH substituent is critical for binding, which is supported by a number of observations:

All chemicals with a 4-OH substituent exhibit binding activity, except 4,4'-methylenebis(2,6-di-tert-butylphenol) that contains four steric hindered tert-butyl groups ortho to the 4- and 4'-OH groups. These prevent $\mathrm{H}$-bond interactions between the phenolic rings and the receptor.

Two benzophenones (Figure 4B), 2,4-dihydroxybenzophenone and 4,4'-dihydroxybenzophenone, both containing a 4-OH group, exhibit weak binding activity. However, 2,2'-dihydroxybenzophenone is inactive due to the absence of a 4-OH group. Similarly, 2,2'-dihydroxy4-methoxybenzophenone and 2-OH-4-methoxybenzophenone are inactive because of the methylation of the $4-\mathrm{OH}$.

Among the 12 DDTs (Figure 4C), the four with 4-OH substitution show much stronger binding than the others. Methoxychlor and methoxychlor olefin are inactive because of methylation of their $\mathrm{OH}$ groups.

Bisphenol A (RBA $=0.008)$ and cumyl phenol $(\mathrm{RBA}=$ 0.005 ) have comparable binding affinities, as do monohydroxymethoxychlor $(\mathrm{RBA}=0.13)$ and HPTE $(\mathrm{RBA}=$ 0.25 ). It suggests that only one phenolic ring is critical in binding for chemicals with the $\mathrm{sp}^{3}$ hybrid bridged carbon. However, this is not the case for chemicals with the $\mathrm{sp}^{2}$ hybrid bridged carbon. Dihydroxymethoxylchlor
Table 4. Hydrophobicity $(\log P)$ of DDTs

\begin{tabular}{|c|c|c|c|c|c|}
\hline DDT & $\log P$ & DDT & $\log P$ & DDT & $\log P$ \\
\hline $0, p^{\prime}$ & 6.7 & $0, p^{\prime}-D D D$ & 5.87 & $0, p^{\prime}-\mathrm{DDE}$ & 6.0 \\
\hline$p, p^{\prime}-D D T$ & 6.91 & $p, p^{\prime}-D D D$ & 6.02 & $p, p^{\prime}-D D E$ & 6.51 \\
\hline
\end{tabular}

ol efin (RBA $=2.47$ ) binds 10 times stronger than monohydroxymethoxychlor ol efin (RBA $=0.23$ ). This suggests that the second phenolic ring of methoxychlor olefin derivatives contributes to the binding, which may be analogous to the $17 \beta-\mathrm{OH}$ of $\mathrm{E}_{2}$. The crystal structures of four ligands with the ER reveal that a considerable flexibility of the $\mathrm{His} 524$ residue of the receptor permits $\mathrm{H}$-bond interactions with ligands in a wide range of relative positions. Because of the $\mathrm{sp}^{2}$ hybridization, the angle between the two phenyls in ol efins $\left(120^{\circ}\right.$ with $\left.\mathrm{sp}^{2}\right)$ are larger than that in the non-ol efin methoxychlors (109 with $\mathrm{sp}^{3}$ ). The distance between the two $\mathrm{O}$ atoms for olefin derivatives is $\sim 9.7 \AA$, and that for the non-olefins is $\sim 9.3 \AA$. Therefore, the $\mathrm{O}-\mathrm{O}$ distance for olefin methoxychlors is closer to that in $E_{2}$ and more favorable for binding.

For diphenolalkanes, changes in chain length at the bridging carbon lead to the RBA changes: bisphenol $B$ $(\mathrm{RBA}=0.086)>$ bisphenol $\mathrm{A}(\mathrm{RBA}=0.008)>$ bisphenol $F(R B A=0.0009)$. The longer the side chain, the greater the binding affinity for the ER (35). Common pharmacophore identification indicated that the optimal structural superposition between bisphenol $A$ and $E_{2}$ is one in which the two benzene rings of bisphenol $A$ are positioned over the $A$ and $C$ rings of $E_{2}$, with the bridge carbon overlaying the $B$ ring (Figure 9). Thus, the effect of steric substituents on the bridge atom of diphenylmethanes on ER binding is analogous to the effect of $7 \alpha$-substituents on $\mathrm{E}_{2}$ binding.

DDT, DDD, and DDE isomers have structural frameworks similar to that of bisphenol A (Figure 4C). The o, $p^{\prime}-$ isomers are active in binding, while $p, p^{\prime}$-isomers are not. The orthochl orine of $0, p^{\prime}$-isomers mi mics the steric $11 \beta$ substituent of $E_{2}$ and increases structural rigidity, which favors binding. DDT isomers have greater hydrophobicity $(\log P)$ than either DDD or DDE isomers (Table 4), which might explain why o,p'-DDT is the strongest binder among the six DDT derivatives that were evaluated.

Biphenyls. 4-OH-PCBs tend to be good binders (Figure 5A), which is consistent with observations in other chemical classes. As the number of chloro substitutions at the nonphenolic ring increases, more electron withdrawal is found in the phenolic ring, which results in higher $\mathrm{pK}_{\mathrm{a}}$ values (36) and a better $\mathrm{H}$-bond donor. $2^{\prime}, 3^{\prime}, 4^{\prime}, 5^{\prime}$-Tetrachloro-4-biphenylol and 2',5'-dichloro-4biphenylol are the strongest binders in the group. Both chemicals have an orthochlorine substitution at the nonphenolic ring. Korach et al. (9) reported that PCB compounds with the strongest affinities possess either single or multi-orthochlorine substitution. The ortho substitution restricts the conformational flexibility of PCBs, which favors binding. However, our data show that a considerable improvement of binding is only associated with the orthochlorine substitution at the nonphenolic ring. For example, 2-chloro-4-bi phenylol $($ RBA $=0.002$ ) has binding affinity similar to that of 4'chloro-4-biphenylol (RBA $=0.007$ ), but 28 times lower than that of 2',5'-dichloro-4-biphenylol (RBA $=0.036$ ). Analyzing common pharmacophores between $E_{2}$ and $2^{\prime}, 3^{\prime}, 4^{\prime}, 5^{\prime}$-tetrachloro-4-biphenylol (Figure 9), we found 

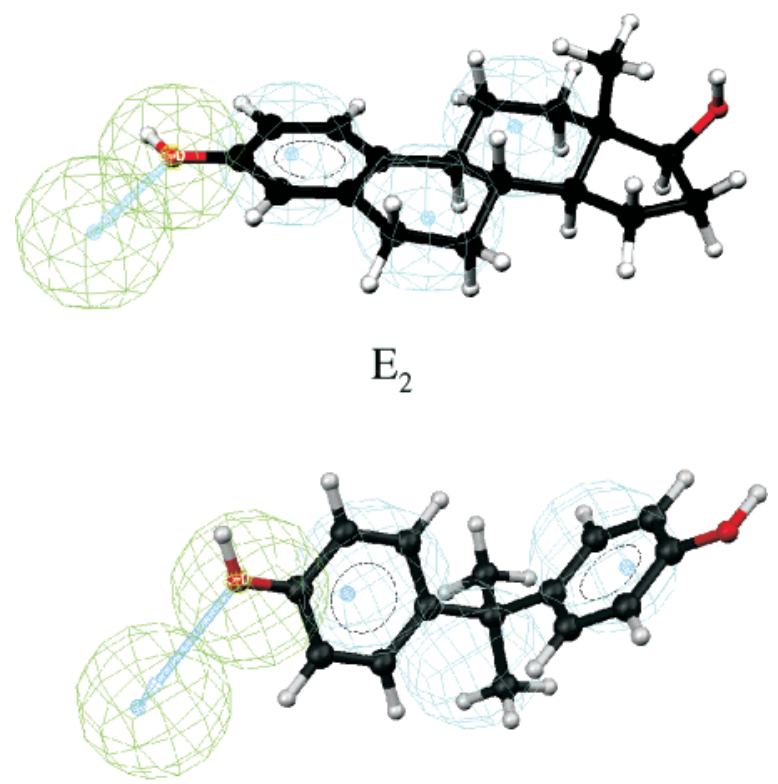

Bisphenol A

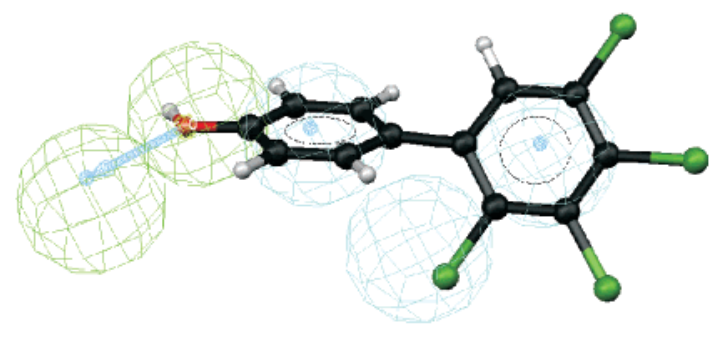

2',3',4',5'-Tetrachloro-4-biphenylol

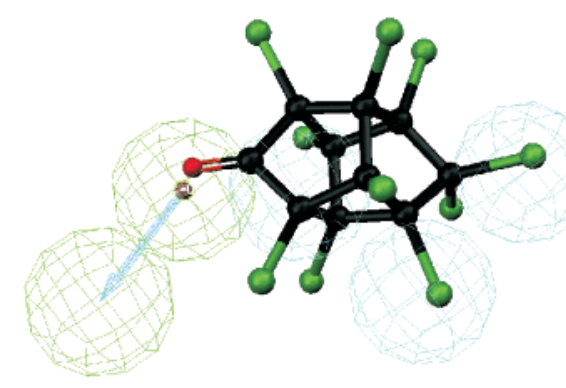

\section{Kepone}

Figure 9. Four-point pharmacophore of $\mathrm{E}_{2}$, the $3-\mathrm{OH}$ and the centers of rings $A, B$, and $C$, identified three structurally diverse xenoestrogens, bi sphenol A, 2', 3', 4', '5'-tetrachloro-4-bi phenylol, and ketone.

that the two benzene rings of $\mathrm{PCBs}$ match the $\mathrm{A}$ and $\mathrm{C}$ ring of $E_{2}$ very well while the orthochlorine occupies the $\mathrm{B}$ ring position. For PCBs having two orthochlorine substitutions at the nonphenolic ring, the second orthochlorine is most likely to overlay the $11 \beta$-position of $\mathrm{E}_{2}$ where the introduction of small steric substituents normally improves binding. Thus, these types of PCBs are better binders. It is evident that $2^{\prime}, 4^{\prime}, 6^{\prime}$-trichloro-4biphenylol is more active than $2^{\prime}, 3^{\prime}, 4^{\prime}, 5^{\prime}$-tetrachloro-4biphenylol (9). It is safe to conclude that the contributions of orthochlorine substitution to binding are associated not only with the restriction of conformational flexibility but

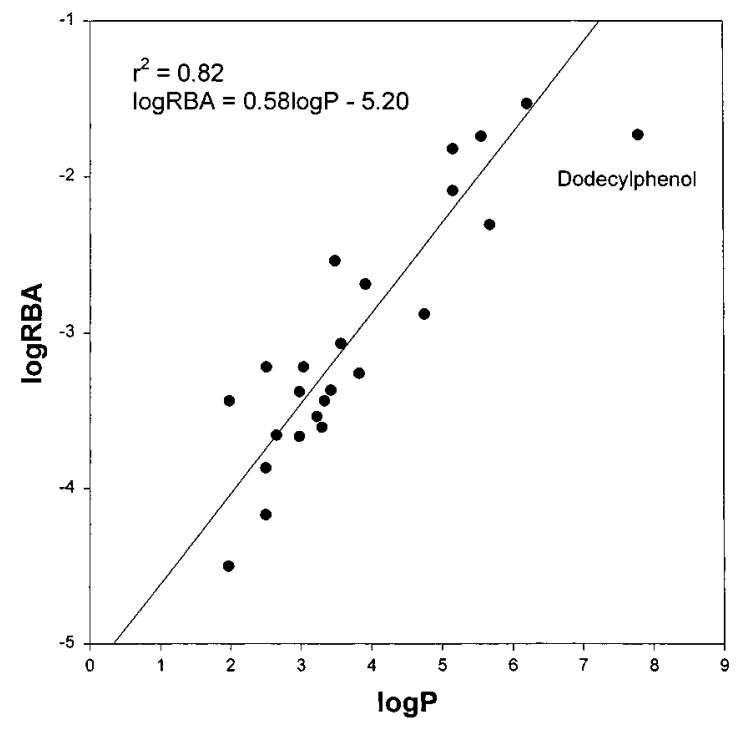

Figure 10. Correlation of $\log$ RBA with $\log P$ for phenols.

also with possibly acting as a hydrophobic group resembling the $\mathrm{B}$ ring and/or $11 \beta$-substituent of $\mathrm{E}_{2}$.

The phenylphenols (Figure 5B) are relatively lower affinity binders than the 4-OH-PCBs. With the change of $\mathrm{OH}$ position from 4 to 3 and to 2, the RBA decreases in the following order due to the decrease in the accessibility of the $\mathrm{OH}$ group for $\mathrm{H}$-bond interaction: 4-phenylphenol > 3-phenylphenol > 2-phenylphenol.

Phenols. Alkyl phenols (Figure 6A), parabens (Figure $6 \mathrm{~B}$ ), and alkyloxyphenols (Figure 6C) are contained in this class; nonyl phenol has the highest RBA (0.031). The activities of the phenols are largely dependent on the alkyl chain length at the para position. 2-Ethylhexylparaben (RBA $=0.018$ ) has an RBA close to that of 4-tert-octyl phenol $(\mathrm{RBA}=0.015)$, and a chain length with the same number of carbons (eight). The activity of parabens follows this order: 2-ethylhexyl > heptyl > benzyl > butyl > propyl = ethyl > methyl. A similar trend is also observed in alkylphenols $(11,23)$. The relationship of ER binding activity ( $\log$ RBA) with hydrophobicity $(\log P)$ is linearly correl ated for this class $\left(r^{2}=0.82, \log R B A=0.58 \log P-5.20 ;\right.$ Figure 10). Each unit of $\log P$ change will cause 0.58 unit of $\log B A$ change, demonstrating the importance of hydrophobicity for binding. However, dodecyl phenol is an outlier, indicating that this linear correlation is only valid within a certain range.

Miscellaneous. A number of miscellaneous chemicals in the NCTR data set were identified to be active in ER binding (Figure 7). Some have similar structural frameworks as previously discussed structural classes, while others do not.

Doisynolic and allenolic acids are nonsteroidal estrogens. Their binding affinities for the ER are normally 100 -fold lower than that of $E_{2}$, but their in vivo potencies are high because of a long duration of action (37). Two of their derivatives, $\alpha, \alpha$-dimethyl- $\beta$-ethylallenolic acid and doisynoestrol, were tested; both exhibited an ability to compete $\mathrm{E}_{2}$ for $\mathrm{ER}$ binding. Their $\mathrm{OH}$ and acid groups as well as their naphthal ene substructures match the 3and $17 \beta-\mathrm{OH}$ and $\mathrm{A}$ and $\mathrm{B}$ rings of $\mathrm{E}_{2}$, respectively, very well in $3 \mathrm{D}$ space. ${ }^{3}$ Because of the methylation of its $\mathrm{OH}$

${ }^{3}$ X. Qian, H. Fang, H. Hong, W. Tong, R. Perkins, and D. M. Sheehan, unpublished results. 
group, doisynoestrol has activity $\sim 100$-fold lower than that of doisynolic acid, which is consistent with the observations for steroids.

4,4'-Methylenedianiline and 4-aminophenyl ether structurally resemble bisphenol $\mathrm{F}$, where the $\mathrm{OH}$ groups are replaced with the amino groups. Both chemicals are inactive in binding. A similar result is also observed for butyl-2-aminobenzoate, which is structurally similar to butyl paraben. This evidence reinforces the importance of the phenolic ring in binding, particularly its essential role for weak estrogens.

Benzil is a selective inducer and a potent in vitro activator of microsomal epoxide hydrolase (38). It is al so used as a photoinitiator for the visiblelight polymerizing resin system that is widely applied in modern dentistry (39). Benzil has a structural resemblance to stilbene and has been studied as a new type of inducer of drugmetabolizing enzyme along with stil bene oxide (40). The 4,4'-hydroxylated benzils share a similar structural construction with the 4,4'-hydroxylated stilbenes: two $\mathrm{OH}$ groups separated by two benzene rings with two $\mathrm{sp}^{2}$ (carbonyl) carbons. Their close structural similarity leads to similar binding affinities found for $2,2^{\prime}, 4,4^{\prime}$ tetrahydroxybenzil (RBA $=0.209)($ Figure 7) and 4,4'dihydroxystilbene (RBA $=0.281$ ) (Figure $2 A)$.

Nordihydroguariaretic acid is an antioxidant for fats and oils in foods. It is a flexible molecule with two catechols separated by four carbons. According to our recent study on common pharmacophore identification of estrogens, ${ }^{3}$ nordihydroguariatic acid shares structural commonalities with $E_{2}$ : its two catechol groups mimic $E_{2}$ 's $A$ and $D$ rings, while its bridged carbons fold in such a way to occupy the space recognized by $7 \alpha$-substituents of $E_{2}$. Normally, hydroxylation of phenol to create catechols reduces estrogenic activity (34), and the flexible structure is not favorable for binding. Therefore, even though nordihydroguariatic acid shares the key pharmacophores of $E_{2}$, its RBA is much lower.

Siloxanes are widely used for industrial and consumer product applications (41). A single subcutaneous injection in mice using samples from breast implant or poly(dimethylsiloxane) results in the wide distribution of the low-molecular weight siloxanes throughout the body (42). Studies by Bennett et al. (43) have shown that a series of low-molecular weight siloxanes alter male reproductive function in a number of mammalian species. An estrogenic effect on the immature rat uterus has also been demonstrated for several organosiloxane compounds (44). Two linear disiloxanes were included for this study. Neither chemical possesses $\mathrm{H}$-bonding capability at either end of the structure which is normally considered to be essential for a chemical to bind to the ER. However, 1,3-diphenyltetramethyldisiloxane exhibits marginal activity. Chal cone (RBA $=0.0015), 1,3$-diphenyltetramethyldisiloxane (RBA $=0.0007$ ), and butylbenzylphthalate (slight binder) share similar structural frameworks: two benzene rings (hydrophobic centers) separated by three atoms. Their activities are proportional to the rigidity of their backbone, suggesting that the precise distance of two hydrophobic centers with a rigid linkage favors binding.

Of the variety of estrogens whose structures have little resemblance to $E_{2}$, kepone is of particular interest for many reasons. Not only does it produce a variety of "estrogen-like" effects on the female reproductive system, but it also causes other toxic effects, including in the neuromuscular system, liver, etc. (45). Kepone (RBA = 0.013 ) was found to be 10000 times weaker than $E_{2}$ in its affinity for the ER. How its structural features relate to $E_{2}$ in binding is still puzzling. Kepone contains four cyclopentanes in the left, right, top, and bottom sides fused together to form a cagelike structure (Figure 7). It appears that the carbonyl group at the $\mathrm{C}_{2}$-position is essential because mirex, its analogue that has the carbonyl group replaced with two chlorine atoms, is inactive in binding. Common pharmacophore identification indicates that kepone is similar to $E_{2}$ in various structural features: the carbonyl group along with the hydrophobic center of the 2,3,3a,5b,1a-cyclopentane ring, $\mathrm{C}_{4}-\mathrm{Cl}$, and the hydrophobic center of the two $\mathrm{Cl}$ atoms at the $\mathrm{C}_{5^{-}}$ position mimics the function of the phenolic $A, B$, and $C$ rings in $E_{2}$ (Figure 9). It is important to point out that kepone can rapidly pick up moisture to form kepone hydrate that should be more active for the ER (46).

\section{Discussion}

In principle, the biological activity of a chemical is determined by its structure. The chemical structure can be represented in three different general ways: 2D substructures, 3D pharmacophores, and physicochemical properties. A 2D substructure is a structural fragment of a molecule, which often can be used as a strong indicator of a particular activity, such as the phenolic ring for the ER. A 3D pharmacophore is a portion of a chemical's 3D structure that is considered essential in eliciting the biological activity of interest, such as the precise $\mathrm{O}-\mathrm{O}$ distance, the orientation of the $\mathrm{OH}$ group, and the location of the hydrophobic center. A physicochemical property of a molecule is a measure of one property of a whole molecule. For example, log P measures a chemical's hydrophobicity. ER binding activity relates to all these structural features. The SAR studies for $E R$ binding by individual chemical classes indicate that some features may well represent binding dependencies for one structural class, while other features may better represent binding dependencies for a different structural class. These structural features are inherently related, suggesting that structural commonality exists among structurally diverse estrogens.

It is well-accepted that the precise spacing of two $\mathrm{OH}$ groups at either end of an essential planar and primarily hydrophobic molecule is considered the structural basis for $E R$ binding (47). The $E_{2}-E R H$-bonding network (Figure 11 ) demonstrates the critical role of $\mathrm{H}$-bonding ability for a chemical to bind to the ER (20). Although the "anchor-like" $\mathrm{H}$-bonding network at the $\mathrm{A}$ ring of $\mathrm{E}_{2}$ imposes an absolute requirement that an effective ligand must contain a phenolic ring, the remainder of the binding pocket can accept a number of different hydrophobic groups. By comparing various estrogen classes through SAR studies, we can summarize the general structural requirements relevant to the template $E_{2}$ structure: (1) $\mathrm{H}$-bonding ability of the phenolic ring mimicking the $3-\mathrm{OH},(2) \mathrm{H}$-bond donor mimicking the $17 \beta-\mathrm{OH}$ and $\mathrm{O}-\mathrm{O}$ distance between 3- and $17 \beta-\mathrm{OH}$, (3) precise steric hydrophobic centers mimicking steric $7 \alpha$ and $11 \beta$-substituents, (4) hydrophobicity, and (5) a ring structure. These important features provide the structural basis for a xenoestrogen to exhibit binding activity.

H-Bonding Ability of the Phenolic Ring Mimicking the 3-OH. The importance of $\mathrm{H}$-bonding has been 


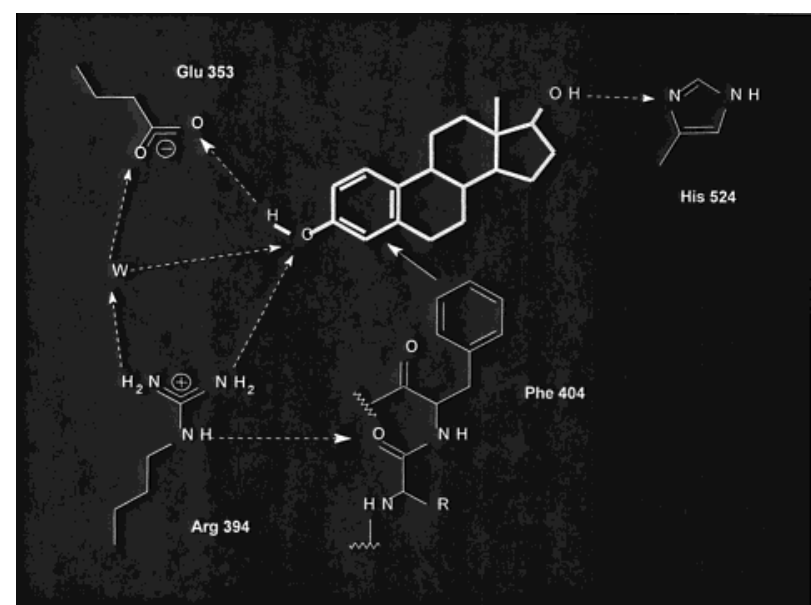

Figure 11. $E R-E_{2}$ hydrogen-bonding network (52).

Table 5. H-Bond Distances between Two OH Groups of Four Ligands and the Receptor

\begin{tabular}{|c|c|c|c|c|}
\hline \multirow[b]{2}{*}{ ER ligand } & \multicolumn{3}{|c|}{$3-\mathrm{OH}$} & $17-\mathrm{OH}$ \\
\hline & $\begin{array}{c}\text { Glu } 353 \\
{\left[\mathrm{~d}_{0-0}(\AA)\right]}\end{array}$ & $\begin{array}{c}\operatorname{Arg} 394 \\
{\left[d_{0-N}(\AA)\right]}\end{array}$ & $\begin{array}{c}\text { water } \\
{\left[\mathrm{d}_{\mathrm{O}-\mathrm{O}}(\AA)\right]}\end{array}$ & $\begin{array}{l}\text { His } 524 \\
{\left[\mathrm{~d}_{\mathrm{O}-\mathrm{N}}(\AA)\right]}\end{array}$ \\
\hline$E_{2}$ & 2.37 & $\begin{array}{l}3.08 \\
332\end{array}$ & $\begin{array}{l}2.82 \\
3.19\end{array}$ & 2.77 \\
\hline $\mathrm{DF}$ & 2.51 & 3.33 & 3.19 & 2.67 \\
\hline raloxifene & 2.38 & 2.93 & 2.97 & 2.62 \\
\hline 4-OH-tamoxifen & 2.45 & 3.04 & 3.07 & \\
\hline
\end{tabular}

recognized in most biological systems. The $\mathrm{O}$ atom is one of the most important heteroatoms directly associated with $\mathrm{H}$-bonding. Of the 130 active chemicals in the NCTR data set, 125 chemicals contain an $\mathrm{O}$ atom and 3 chemicals contain a $\mathrm{Cl}$ atom that may serve as a weak $\mathrm{H}$-bond acceptor. Even though the degree of specificity is reduced because of two active chemicals without $\mathrm{H}$-bonding ability that produce specific steric effects, it is difficult to avoid the conclusion that the requirement of $\mathrm{H}$-bonding ability is the most important characteristic for estrogen.

It has been long understood that the phenolic ring is normally associated with estrogenic activity. One hundred eight of 130 active chemicals (83\%) in the NCTR data set contain a phenolic ring. The contribution of the phenolic ring in binding is much more significant than any other structural feature. The current crystal structures of four ligand-ER complexes reveal that the phenolic rings of all four ligands are closely positioned at the same location to allow $\mathrm{H}$-bond interactions with Glu 353, Arg 394 of the receptor, and a water molecule. The distance of the phenolic $\mathrm{O}$ atom of these four ligands to Glu 353, Arg 394, and the water molecule is summarized in Table 5. It appears that the $\mathrm{H}$-bonding of the phenolic OH is much stronger with Glu 353 than Arg 394 and the water molecule because of the shorter distance. Thus, the phenolic $\mathrm{OH}$ contributes to binding largely through its $\mathrm{H}$-bond donor ability because of the $\mathrm{H}$-bond acceptor properties of Glu 353. The phenolic $\mathrm{OH}$ is a better $\mathrm{H}$-bond donor than an acceptor. Replacing $\mathrm{OH}$ with the weak $\mathrm{H}$-bond donor $\mathrm{NH}_{2}$ reduces activity for $\mathrm{E}_{2}$ (17), and diminishes activity for bisphenol $\mathrm{F}$ and butylparaben (4-aminophenyl ether and butyl-4-aminobenzoate, Figure 7). It is important to point out that the $\mathrm{H}$-bond donor ability of the phenolic $\mathrm{OH}$ is dependent on a number of factors, particularly the nature of ortho substituents that affect the $\mathrm{OH}$ accessibility. The $\mathrm{H}$-bond donor ability for several ortho-substituented phenols follows this trend: phenol $>$ 2-methylphenol $=2$-tert-butylphenol $>2,6$ dimethylphenol > 2,6-di-tert-butylphenol (48), in which 2,6-di-tert-butylphenol is not a $\mathrm{H}$-bond donor. This is consistent with the lack of binding activity observed for 4,4'-methylenebis(2,6-di-tert-butyl phenol) (Figure 4A). In conclusion, a good $\mathrm{H}$-bond donor mimicking the $3-\mathrm{OH}$ of $\mathrm{E}_{2}$ may be more important than a $\mathrm{H}$-bond acceptor for xenoestrogen.

$\mathrm{H}-\mathrm{Bond}$ Donor Mimicking the $17 \beta-\mathrm{OH}$ and $\mathrm{O}-\mathrm{O}$ Distance between 3- and $17 \beta-O H$. Chemicals could be strong estrogens if they possess a phenolic ring and an additional $\mathrm{OH}$ group within a certain distance range. Chemicals containing only one phenolic group are most likely to be weak to medium ER ligands. The $17 \beta-\mathrm{OH}$ of $\mathrm{E}_{2}$ makes a single $\mathrm{H}$-bond as a donor with $\mathrm{H}$ is 524 , because the two $\mathrm{N}$ atoms of imidazole act as an $\mathrm{H}$-bond acceptor in most cases. This is consistent with the observation of similar RBAs for the $\mathrm{E}_{1}$ and 17-deoxy- $\mathrm{E}_{2}$ (Figure 1A) pair and the meso-p-( $\alpha, \beta$-diethyl-p-methylphenethyl)phenol and hexestrol monomethyl ether pair (Figure $2 \mathrm{~B}$ ). Therefore, a stronger $\mathrm{H}$-bond donor, such as the phenolic $\mathrm{OH}$, favors binding at the position that resembles the $17 \beta-\mathrm{OH}$ of $\mathrm{E}_{2}$.

The imidazole of His 524 is not only able to rotate (because of the alkyl chain), but its two equivalent imidazole $\mathrm{N}$ atoms could compensate for the change in the oxygen position of $17 \beta-\mathrm{OH}$ and maintain a favorable $\mathrm{H}$-binding position. This was recognized in the $\mathrm{E}_{2}$-and raloxifene-ER crystal structure. The phenolic A rings of both molecules overlap quite well, whereas the $17 \beta-\mathrm{OH}$ group of $\mathrm{E}_{2}$ and the $\mathrm{OH}$ group of raloxifene are $5 \AA$ apart. However, these two $\mathrm{OH}$ groups have a similar distance to the $\mathrm{N}$ atom of the imidazole (Table 5). It suggests that the flexible $\mathrm{His} 524$ is more tolerant of the second $\mathrm{OH}$ groups of a chemical in forming an effective $\mathrm{H}$-bond interaction. Although the $\mathrm{H}$-bonding strength is dependent on the relative positions (angle and distance) between the imidazole of $\mathrm{His} 524$ and the $\mathrm{OH}$ group, the $\mathrm{O}-\mathrm{O}$ distance could provide a fair estimation of the $\mathrm{H}$-bonding ability of the second $\mathrm{OH}$ group analogous to the $17 \beta-\mathrm{OH}$ of $E_{2}$.

For purposes of analysis, we have divided the NCTR data set into four activity categories: strong (RBA $>1)$, medium $(1>$ RBA > 0.01), weak $(0.01>$ RBA > detectable activity), and inactive. As shown in Figure 12, most strong to medium ER ligands contain two $\mathrm{OH}$ groups with an $\mathrm{O}-\mathrm{O}$ distance ranging from 9.7 to 12.3 $\AA$. The chemicals with an $\mathrm{O}-\mathrm{O}$ distance not in this range are highly likely to be weak estrogens.

Precise Steric Hydrophobic Centers Mimicking Steric $7 \alpha$ - and $\mathbf{1 1} \beta$-Substituents. The volume of the $E R$ binding pocket $\left(450 \AA^{3}\right)$ is about twice that of $E_{2}\left(245 \AA^{3}\right)$ (20). The length and breadth of the $E_{2}$ skeleton are well matched by the receptor, but there are large unoccupied cavities at the $7 \alpha$ - and $11 \beta$-positions of $E_{2}$. The positions of these cavities allow steric groups of certain sizes to fit, and are of great importance for xenoestrogens, including DES-like chemicals, diphenylmethanes, and biphenyls. For example, the binding affinities of DES, DMS, and 4,4'-dihydroxystil bene change dramatically with changes in the side chain length on the $\mathrm{C}-\mathrm{C}$ double bond. This trend was also observed (4) in in vivo activity; 4,4'-dihydroxystilbene had 250-fold lower potency than DMS and 16700-fold lower potency than DES. Even triphenylethylene $(\mathrm{RBA}=0.002)$ (Figure 2C) that has no $\mathrm{H}$-bonding ability still exhibits weak 


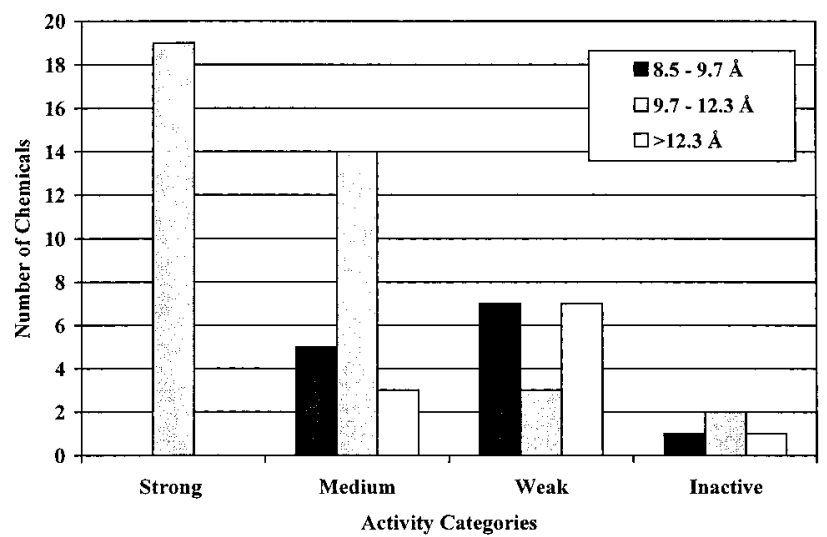

Figure 12. Effect of the range of the $\mathrm{O}-\mathrm{O}$ distance on RBA. The activity categories were arbitrarily defined as follows. F or strong estrogens, RBA $>1$. For medium estrogens, the RBA is between 0.01 and 1 . For the weak estrogens, RBA is between 0.01 and a detectable activity. Inactive chemicals show no detectable activity in the assay. The RBA for $E_{2}$ is set to 100 .

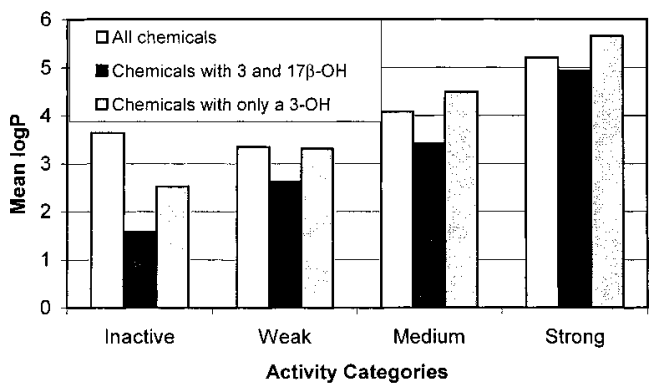

Figure 13. Relationship of the mean $\log P$ values with binding activity. The activity categories are defined in the legend of Figure 12

binding. Clearly, the precise location of steric bulk in the region of the $7 \alpha$-and/or $11 \beta$-positions of $\mathrm{E}_{2}$ is critical for binding. It is evident that DL-hexestrol's RBA activity is about 100-fold lower than that of mesohexestrol. The two isomers only differ in the direction of the two ethyl substitutes. Similarly, indenestrol A was found to have higher affinity for the ER (49) than the enantiomers of indenestrol B (50).

Hydrophobicity. The ER ligand-binding pocket has a 3D "cross"-like shape; the center and vertical ends are mainly hydrophobic, whereas the polar functions are located at opposite ends of the horizontal cavity. An effect of hydrophobicity in binding was observed for phthalates (51). Hydrophobicity $(\log P)$, expressed as a ratio of solubility between octanol and water, is important for many biological end points. Log $\mathrm{P}$ values too high or too low can be associated with poor transport characteristics. Unlike the aforementioned three estrogen-related structural features that are critical in binding only by interacting with specific amino acids of the receptor, hydrophobicity is a physicochemical property.

Although the mean log P values for strong, medium, and weak estrogens exhibited a positive trend (Figure 13), the inactive chemicals also have a wide range of $\log P$ values. This suggests that better binders tend to have larger log $P$ values but not vice versa. We plotted $\log$ RBA versus $\log$ P for individual chemical classes; the only linear correlation was for phenols (Figure 10). Phenols consist of only a phenolic ring serving as a $\mathrm{H}$-bond anchor and an alkyl chain for hydrophobicity. The linear correlation between $\log$ RBA and $\log$ P for phenols suggests that $\log P$ is only important in binding when the key pharmacophores are in place. This can be further demonstrated by plotting mean log $\mathrm{P}$ values versus activity categories for chemicals containing two $\mathrm{OH}$ groups with distances between 9.7 and $12.3 \AA$ (mimicking the 3- and $17 \beta-\mathrm{OH}$ of $\mathrm{E}_{2}$ ) and only one $3-\mathrm{OH}$ group. As shown in Figure 13, the positive effect of hydrophobicity on binding is much more apparent for chemicals containing the 3- and $17 \beta-\mathrm{OH}$, and a $3-\mathrm{OH}$. Interestingly, for each activity category, the mean $\log \mathrm{P}$ value of chemicals with one $\mathrm{OH}$ group is larger by almost the same amount compared to that of chemicals containing two $\mathrm{OH}$ groups mimicking the 3- and $17 \beta-\mathrm{OH}$ of $\mathrm{E}_{2}$. This indicates that chemicals lacking the effective $\mathrm{O}-\mathrm{O}$ distance require greater hydrophobicity to reach binding activities similar to those of chemicals with the $3-$ and $17 \beta-\mathrm{OH}$ groups.

Ring Structure. Effective ER ligands must possess a ring structure. A literature survey of more than 2000 unique chemicals that were tested by various in vitro and/or in vivo assays for estrogenic activity reveals that no estrogenic chemical has been found that does not possess a ring structure. The ring construction increases the rigidity of both the structure and the steric center, which favors ER binding. It appears that the flat aromatic ring, which fits better to the narrow "tunnel-like" arrangement in the receptor for the phenolic A ring, is more important than other ring structures. The majority of xenoestrogens contain at least one aromatic ring. Only five chemicals without aromatic rings were found to be active, of which four are steroids (Figure 1B) in addition to kepone. All five chemicals possess $\mathrm{H}$-bond capability with a rigid hydrophobic backbone that matches the $A$, $B$, and $C$ rings of $E_{2}$ very well. It suggests that nonaromatic estrogens require several key structural features to exhibit binding activity, at least including a strong electronegative atom ( $\mathrm{O}, \mathrm{S}, \mathrm{N}$, etc.) for $\mathrm{H}$-bond interaction and a rigid hydrophobic backbone.

There are several substructures inherently associated with ER binding activity. The common structural framework for most estrogens contains an aromatic ring, normally a phenolic ring, separated from a hydrophobic center that mimics one of the centers of $E_{2}$ 's $B, C$, or $D$ ring. PCBs have two benzene rings connected directly, which resemble the $A$ and $C$ rings. The ortho substituents of $\mathrm{PCBs}$ match the $\mathrm{B}$ ring and/or $11 \beta$-substituents of $\mathrm{E}_{2}$ and increase binding affinity. Bisphenol A and DDT-like chemicals have two benzene rings separated by one atom, whereas two rings mimic the $A$ and $C$ ring and the substituents on the bridge atom match the $B$ ring. The two benzene rings of DES derivatives resemble the $A$ and $D$ rings. The binding of DES derivatives is mainly governed by the presence or absence of two $\mathrm{OH}$ groups at the 4,4'-position and two ethyl groups to mimic the $7 \alpha$ - and $11 \beta$-substituents of $\mathrm{E}_{2}$. It seems that two benzene rings separated by three atoms provide an optimal steric interaction with the receptor, which is demonstrated by three weak estrogens, chal cone, 1,3-diphenyltetramethyldisiloxane, and butylbenzylphthalate, which contain no $\mathrm{H}$-bonding capability mimicking either the 3 - or $17 \beta-\mathrm{OH}$ of $\mathrm{E}_{2}$.

Summary. The SAR studies on 230 diverse chemicals reveal five structural features that are most important for chemical binding to $E R$. The representative chemical for each structural category listed in Table 1 demonstrates that the more key structural features a chemical contains, the more active it is. These findings can be 


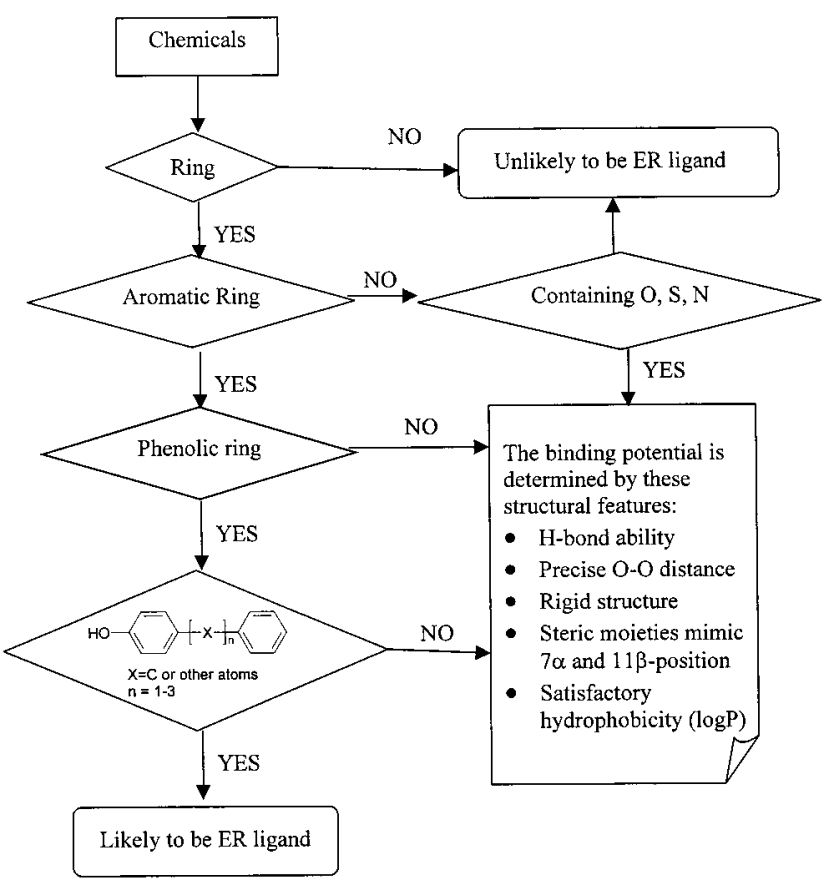

Figure 14. Flowchart for identification of ER ligands.

generalized as a set of "if-then" rules for guidance in identifying potential ER ligands, which is depicted in Figure 14:

(1) If a chemical contains no ring structure, then it is unlikely to be an ER ligand.

(2) If a chemical has a nonaromatic ring structure, then it is unlikely to be an ER ligand if it does not contain an $\mathrm{O}, \mathrm{S}, \mathrm{N}$, or other heteroatom for $\mathrm{H}$-bonding. Otherwise, its binding potential is dependent on the existence of the key structural features. Kepone, dihydrotestosterone, norethynodrel, and $3 \alpha$ - and $3 \beta$-androstanediol are active ER ligands that fall into this category.

(3) If a chemical has a non-OH aromatic structure, then its binding potential is dependent on the existence of the key structural features. A total of 16 chemicals in the NCTR data set, including o,p'-DDT, 1,3-diphenyltetramethyldisiloxane, 3-deoxyl-E $E_{2}$ mestranol, and others, fall into this category.

(4) If a chemical contains a phenolic ring, then it tends to be an ER ligand if it contains any additional key structural features. For the chemicals containing a phenolic ring separated from another benzene ring with the number of bridge atoms ranging from none to three, it will most likely be an ER ligand.

\section{Conclusion}

The goal of the study presented here was to explore the change in ER binding activity with the change of structure based on the NCTR data set, which to the best of our knowledge is so far the largest published structurally diverse data set with a consistent quantitative binding activity measurement. The data set conveys information far beyond the scope of this paper. The knowledge derived from this study enhances our understanding of the important ER binding-related structural features, which in turn should be important in guiding the development of predictive toxicology models for rapid identification of xenoestrogens using computational approaches. No matter what computational approaches are used, the primary step is to identify ER-related structural characteristics. The knowledge then could be used to design alignment for 3D QSAR, to select ER-related structural descriptors for QSAR, and to identify key structural features for pharmacophore models. All this tells us that structure-activity relationship analyses of xenoestrogens are essential.

Acknowledgment. We gratefully acknowledge the U.S. Department of Energy and the U.S. Food and Drug Administration (FDA) for postdoctoral support through the Oak Ridge I nstitute for Science and E ducation. This work was partially supported by the FDA's Office of Women's Health and the Chemical Manufacturer's Association.

\section{References}

(1) Kavlock, R. J ., Daston, G. P., DeRosa, C., Fenner-Crisp, P., Gray, L. E., Kaattari, S., Lucier, G., Luster, M., Mac, M. J ., Maczka, C., Miller, R., Moore, J ., Rolland, R., Scott, G., Sheehan, D. M., Sinks, T., and Tilson, H. A. (1996) Research needed for the risk assessment of health and environmental effects of endocrine disruptors: a report of the U.S. EPA-sponsored workshop. Environ. Health Perspect. 104 (Suppl. 4), 715-740.

(2) Patlak, M. (1996) A testing deadline for endocrine disrupters. Environ. Sci. Technol. 30, 540A-544A.

(3) Katzenellenbogen, J. A. (1995) The structural pervasiveness of estrogenic activity. Environ. Health Perspect. 103 (Suppl. 7) 99-101.

(4) Dodds, E., Goldberg, L., and Lawson, W. (1938) Oestrogenic activity of alkylated stilboestrols. Nature 142, 34

(5) Dodds, E., and Lawson, W. (1938) Molecular structure in relation to oestrogenic activity. Compounds without a phenanthrene nucleus. Proc. R. Soc. 125, 222-232.

(6) Anstead, G. M., Carlson, K. E., and Katzenellenbogen, J . A. (1997) The estradiol pharmacophore: ligand structure-estrogen receptor binding affinity relationships and a model for the receptor binding site. Steroids 62, 268-303.

(7) Dodge, J., and J ones, C. (1999) Non-steroidal estrogens. In Estrogens and antiestrogens I: Physiology and Mechanisms of Avtion of estrogens and antiestrogens (Oettel, M., and Schillinger, E., Eds.) pp 43-53, Springer-Verlag, Berlin.

(8) Sadler, B. R., Cho, S. J ., I shaq, K. S., Chae, K., and Korach, K. S (1998) Three-dimensional quantitative structure-activity relationship study of nonsteroidal estrogen receptor ligands using the comparative molecular field analysis/cross-validated r2-guided region selection approach. J . Med. Chem. 41, 2261-2267.

(9) Korach, K., Sarver, P., Chae, K., McLachlan, J ., and McKinney, J . (1988) Estrogen receptor-binding activity of polychlorinated hydroxybiphenyls: conformationally restricted structural probes. Mol. Pharmacol. 33, 120-126.

(10) Miksicek, R. J . (1994) Interaction of naturally occurring nonsteroidal estrogens with expressed recombinant human estrogen receptor. J . Steroid Biochem. Mol. Biol. 49, 153-160.

(11) Routledge, E. J ., and Sumpter, J . P. (1997) Structural features of alkyl phenolic chemicals associated with estrogenic activity. J Biol. Chem. 272, 3280-3288.

(12) Grese, T. A., Cho, S., Finley, D. R., Godfrey, A. G., J ones, C. D., Lugar, C. W., III, Martin, M. J ., Matsumoto, K., Pennington, L. D., Winter, M. A., Adrian, M. D., Cole, H. W., Magee, D. E., Phillips, D. L., Rowley, E. R., Short, L. L., Glasebrook, A. L., and Bryant, H. U. (1997) Structure-activity relationships of selective estrogen receptor modulators: modifications to the 2-arylbenzothiophene core of raloxifene. J . Med. Chem. 40, 146-167.

(13) Tong, W., Perkins, R., Strelitz, R., Collantes, E. R., Keenan, S., Welsh, W. J., Branham, W. S., and Sheehan, D. M. (1997) Quantitative structure-activity relationships (QSARs) for estrogen binding to the estrogen receptor: predictions across species. Environ. Health Perspect. 105, 1116-1124.

(14) Waller, C. L., Oprea, T. I., Chae, K., Park, H. K., Korach, K. S., Laws, S. C., Wiese, T. E., Kelce, W. R., and Gray, L. E., J r. (1996) Ligand-based identification of environmental estrogens. Chem. Res. Toxicol. 9, 1240-1248.

(15) Tong, W., Perkins, R., Xing, L., Welsh, W. J ., and Sheehan, D. M. (1997) QSAR models for binding of estrogenic compounds to estrogen receptor $\alpha$ and $\beta$ subtypes. Endocrinology $\mathbf{1 3 8}$ $4022-4025$ 
(16) Tong, W., Lowis, D. R., Perkins, R., Chen, Y., Welsh, W. J . Goddette, D. W., Heritage, T. W., and Sheehan, D. M. (1998) Evaluation of quantitative structure-activity relationship methods for large-scale prediction of chemicals binding to the estrogen receptor. J. Chem. Inf. Comput. Sci. 38, 669-677.

(17) Wiese, T. E., Polin, L. A., Palomino, E., and Brooks, S. C. (1997) Induction of the estrogen specific mitogenic response of MCF-7 cells by selected analogues of estradiol-17 beta: a 3D QSAR study. J. Med. Chem. 40, 3659-3669.

(18) Gantchev, T. G., Ali, H., and van Lier, J. E. (1994) Quantitative structure-activity relationships/comparative molecular field analysis (QSAR/CoMFA) for receptor-binding properties of hal ogenated estradiol derivatives. J. Med. Chem. 37, 4164-4176.

(19) Waller, C. L., Minor, D. L., and McKinney, J . D. (1995) Using three-dimensional quantitative structure-activity relationships to examine estrogen receptor binding affinities of polychlorinated hydroxybiphenyls. Environ. Health Perspect. 103, 702-707.

(20) Brzozowski, A. M., Pike, A. C., Dauter, Z., Hubbard, R. E., Bonn, T., Engstrom, O., Ohman, L., Greene, G. L., Gustafsson, J. A., and Carlquist, M. (1997) Molecular basis of agonism and antagonism in the oestrogen receptor. Nature 389, 753-758.

(21) Shiau, A. K., Barstad, D., Loria, P. M., Cheng, L., Kushner, P. J ., Agard, D. A., and Greene, G. L. (1998) The structural basis of estrogen receptor/coactivator recognition and the antagonism of this interaction by tamoxifen. Cell 95, 927-937.

(22) Shi, L. M., Fang, H., Tong, W., Wu, J., Perkins, R., Blair, R., Branham, W., and Sheehan, D. (2001) QSAR models using a Iarge diverse set of estrogens. J . Chem. Inf. Comput. Sci. 41 (1), 186-195.

(23) Blair, R., Fang, H., Branham, W. S., Hass, B., Dial, S. L., Moland C. L., Tong, W., Shi, L., Perkins, R., and Sheehan, D. M. (2000) Estrogen Receptor Relative Binding Affinities of $188 \mathrm{Natural}$ and Xenochemicals: Structural Diversity of Ligands. Toxicol. Sci. 54, 138-153.

(24) Branham, W. S., Dial, S. L., Moland, C. L., Hass, B., Blair, R., Fang, H., Shi, L., Tong, W., Perkins, R., and Sheehan, D. M. (2001) Phytoestrogen and Mycoestrogen Binding to Rat Uterine Estrogen Receptor. Am. J . Nutr. (in press).

(25) Fang, H., Tong, W., Perkins, R., Soto, A., Prechtl, N., and Sheehan, D. M. (2000) Quantitative comparison of in vitro assays for estrogenic activity. Environ. Health Perspect. 108, 723-729.

(26) Zacharewski, T. (1998) I dentification and assessment of endocrine disruptors: limitations of in vivo and in vitro assays. Environ. Health Perspect. 106 (Suppl. 2), 577-582.

(27) Meylan, W., and Howard, P. (1995) Atom/Fragment Contribution Method for Estimating Octanol-Water Partition Coefficients. J. Pharm. Sci. 84, 83-92.

(28) Von Angerer, E. (1995) in The Estrogen Receptor As Target For Rational Drug Design (von Angerer, E., Ed.) p 36, R. G. Landes Co., Austin, TX.

(29) Bucourt, R., Vignau, M., and Torelli, V. (1978) New biospecific adsorbents for the purification of estradiol receptor. J . Biol. Chem. 253, 8221-8228

(30) J ordan, V. C., Mittal, S., Gosden, B., Koch, R., and Lieberman M. E. (1985) Structure-activity relationships of estrogens. Environ. Health Perspect. 61, 97-110.

(31) Pike, A., Brzozowski, A., Hubbard, R., Bonn, T., Thorsell, A., Engstrom, O., Ljunggren, J ., Gustafsson, J ., and Carlquist, M (1999) Structure of the ligand-binding domain of oestrogen receptor $\beta$ in the presence of a partial agonist and a full antagonist. EMBO J . 18, 4608-4618.

(32) Nishirara, T., Nishikawa, J ., Kanayama, T., Dakeyama, F., Saito, K., Imagawa, S., Takatori, S., Kitagawa, Y., Hori, S., and Utsumi, H. (2000) Estrogenic Activities of 517 Chemicals by Yeast TwoHybrid Assay. J. Health Sci. 46, 282-298.

(33) Anstead, G. M., Wilson, S. R., and Katzenellenbogen, J. A (1989) 2-Arylindenes and 2-arylindenones: Molecular structures and considerations in binding orientation of unsymmetrical nonsteroidal ligands to the estrogen receptor. J . Med. Chem. 32, 2163-2172.

(34) Miksicek, R. J . (1995) Estrogenic flavonoids: structural requirements for biological activity. Proc. Soc. Exp. Biol. Med. 208, 44-50.
(35) Perez, P., Pulgar, R., Olea-Serrano, F., Villalobos, M., Rivas, A., Metzler, M., Pedraza, V., and Olea, N. (1998) The estrogenicity of bisphenol A-related diphenylalkanes with various substituents at the central carbon and the hydroxy groups. Environ. Health Perspect. 106, 167-174.

(36) Ebner, K., and Braselton, W. (1985) Structural and chemical requirements of hydroxylated polychlorinated biphenyls $(\mathrm{PCBOH})$ for inhibition of energy-dependent swelling of rat liver mitochondria. Toxi col ogist 5, 260.

(37) Scribner, A. W., J onson, S. D., Welch, M. J ., and Katzenellenbogen, J. A. (1997) Synthesis, estrogen receptor binding, and tissue distribution of [ $\left.{ }^{18} \mathrm{~F}\right]$ fluorodoisynolic acids. Nucl. Med. Biol. 24, 209-224.

(38) Seidegard, J., and DePierre, J. W. (1981) Benzil, a selective inducer and a potent in vitro activator of microsomal epoxide hydrolase. Adv. Exp. Med. Biol. 136, 717-728.

(39) Fujisawa, S., Kadoma, Y., and Masuhara, E. (1986) Effects of photoinitiators for the visible-light resin system on hemolysis of dog erythrocytes and lipid peroxidation of their components. J. Dent. Res. 65, 1186-1190.

(40) Seidegard, J ., DePierre, J . W., Morgenstern, R., Pilotti, A., and Ernster, L. (1981) Induction of drug-metabolizing systems and related enzymes with metabolites and structural analogues of stilbene. Biochim. Biophys. Acta 672, 65-78.

(41) McKim, J . M., J r., Choudhuri, S., Wilga, P. C., Madan, A., BurnsNaas, L. A., Gallavan, R. H., Mast, R. W., Naas, D. J ., Parkinson, A., and Meeks, R. G. (1999) Induction of hepatic xenobiotic metabolizing enzymes in female Fischer-344 rats following repeated inhalation exposure to decamethylcyclopentasiloxane (D5) Toxicol. Sci. 50, 10-19.

(42) Kala, S. V., Lykissa, E. D., Neely, M. W., and Lieberman, M. W. (1998) Low molecular weight silicones are widely distributed after a single subcutaneous injection in mice. Am. J. Pathol . 152 645-649.

(43) Bennett, D. R., Gorzinski, S. J., and LeBeau, J. E. (1972) Structure-activity relationships of oral organosiloxanes on the male reproductive system. Toxicol. Appl. Pharmacol. 21, 55-67.

(44) Hayden, J. F., and Barlow, S. A. (1972) Structure-activity relationships of organosiloxanes and the female reproductive system. Toxicol. Appl. Pharmacol. 21, 68-79.

(45) Guzelian, P. S. (1982) Comparative toxicology of chlordecone (kepone) in humans and experimental animals. Annu. Rev. Pharmacol. Toxicol. 22, 89-113.

(46) Wilson, N. K ., and Zehr, R. D. (1979) Structures of some kepone photoproducts and related chlorinated pentacyclodecanes by carbon-13 and proton nuclear magnetic resonance. J . Org. Chem. 44, 1278-1282.

(47) Keasling, H., and Schueler, F. (1950) The relationship between estrogenic action and chemical constitution in a group of azomethine derivatives. J. Am. Pharm. Assoc. 39, 87-90.

(48) Abraham, M., Duce, P., and Prior, D. (1989) Hydrogen Bonding. Part 9. Solute Proton Donor and Proton Acceptor Scales for Use in Drug Design. J. Chem. Soc., Perkin Trans. 2, 1355-1375.

(49) Korach, K. S., Chae, K., Levy, L. A., Duax, W. L., and Sarver, P. . . (1989) Diethylstilbestrol metabolites and analogs. Stereochemical probes for the estrogen receptor binding site. J . Biol. Chem. 264, 5642-5647.

(50) Duax, W. L., Griffin, J. F., Weeks, C. M., and Korach, K. S. (1985) Molecular conformation, receptor binding, and hormone action of natural and synthetic estrogens and antiestrogens. Environ. Health Perspect. 61, 111-121.

(51) Nakai, M., Tabira, Y., Asai, D., Yakabe, Y., Shimyozu, T., Noguchi, M., Takatsuki, M., and Shimohigashi, Y. (1999) Binding characteristics of dialkyl phthalates for the estrogen receptor. Biochem. Biophys. Res. Commun. 254, 311-314.

(52) Tanenbaum, D. M., Wang, Y., Williams, S. P., and Sigler, P. B. (1998) Crystallographic comparison of the estrogen and progesterone receptor's ligand binding domains. Proc. Natl. Acad. Sci. U.S.A. 95, 5998-6003.

\section{TX000208Y}

